8

\title{
Deciphering the interstrand crosslink DNA repair network expressed by Trypanosoma brucei
}

\author{
Ambika Dattani" and Shane Wilkinson* \\ School of Biological and Chemical Sciences, Queen Mary University of London, London, E1 \\ 4NS UK. \\ ${ }^{\#}$ Current address: School of Life Sciences, Queen's Medical Centre, University of Nottingham, \\ Nottingham, UK. \\ *Corresponding author. \\ Email: s.r.wilkinson@qmul.ac.uk
}

Short title: Interstrand crosslink repair in trypanosomes. 


\section{Abstract}

Interstrand crosslinks (ICLs) represent a highly toxic form of DNA damage that can block essential biological processes including DNA replication and transcription. To combat their deleterious effects all eukaryotes have developed cell cycle-dependent repair strategies that coopt various factors from 'classical' DNA repair pathways to resolve such lesions. Here, we report that Trypanosoma brucei, the causative agent of African trypanosomiasis, possesses such systems that show some intriguing differences to those mechanisms expressed in other organisms. Following the identification of trypanosomal homologues encoding for CSB, EXO1, SNM1, MRE11, RAD51 and BRCA2, gene deletion coupled with phenotypic studies demonstrated that all the above factors contribute to this pathogen's ICL REPAIRtoire with their activities split across two epistatic groups. We show that one network, which encompasses TbCSB, TbEXO1 and TbSNM1, may operate throughout the cell cycle to repair ICLs encountered by transcriptional detection mechanisms while the other relies on homologous recombination enzymes that together may resolve lesions responsible for the stalling of DNA replication forks. By unravelling and comparing the T. brucei ICL REPAIRtoire to those systems found in its host, targets amenable to inhibitor design may be identified and could be used alongside trypanocidal ICL-inducing agents to exacerbate their effects.

\section{Author summary}

Parasites belonging to the Trypanosoma brucei complex cause a human and animal infections collectively known as African trypanosomiasis. Drugs used against these diseases are problematic as medical supervision is required for administration, they are costly, have limited efficacy, may cause unwanted side effects while drug resistance is emerging. Against this backdrop, there is a need for new therapies targeting these neglected tropical diseases. Previous studies have shown compounds that induce DNA interstrand crosslinks (ICLs) formation are effective trypanocidal agents with the most potent invariably functioning as prodrugs. Despite the potential of ICL-inducing compounds to treat African trypanosomiasis little is known about the ICL repair mechanisms expressed by trypanosomes. Using a combination of gene deletion and epistatic analysis we report the first systematic dissection of how ICL repair might operate in T. brucei, a diverged eukaryote. It sheds light on the conservation and divergence of ICL repair in one of only a handful of protists that can be studied genetically, and offers the promise of developing or exploiting ICL-causing agents as 
new anti-parasite therapies. These findings emphasise the novelty and importance of understanding ICL repair in T. brucei and, more widely, in non-model eukaryotes.

\section{Introduction}

Spread by the hematophagous feeding behaviour of tsetse flies, protozoan parasites belonging to the Trypanosoma brucei species complex are responsible for a group of human and animal infections collectively known as African trypanosomiasis [1]. In terms of their medical importance, these pathogens have caused several major epidemics across sub-Saharan Africa with the last ending at the turn of the millennium [2]. Based on concerted efforts by the World Health Organisation (WHO), national control programmes and non-governmental organisations, improved screening and new treatment approaches have been implemented resulting in a drastic reduction in the estimated number of cases of human African trypanosomiasis (HAT) from around 300,000 in 1998 to below 20,000 in 2015 [1]. Based on the sustained success of such schemes WHO aims to eliminate the human form of the disease as a public health problem by 2020 , a goal that is now tantalisingly close [3, 4].

Despite significant progress being made to eradicate HAT, a potential drawback surrounds the therapies being used as each have their own problems; some have limited efficacy, others elicit unwanted side effects, most require prolonged periods of hospitalisation while many are expensive to synthesize, transport and/or store [5, 6]. Additionally, parasite strains refractory to treatment have been generated in the laboratory with some of the resistance mutations noted in these lines also observed in the field [7-12]. Together these issues could potentially derail WHOs ambition for HAT elimination. One approach that may help overcome such problems involves determining the mechanism of action for these existing therapies to exploit any findings in the development of new trypanocides. Applying this strategy to nifurtimox, one of the components in the clinically used nifurtimox eflornithine combination therapy, revealed that a mitochondrial NADH dependent type I nitroreductase, an enzyme commonly found in bacteria and some lower eukaryotes but absent from higher eukaryotes, plays a key role in catalysing the conversion of the 5-nitrofuran prodrug to its cytotoxic products [13]. Screening programmes utilizing this activity has identified a range of anti-T. brucei compounds. This revealed that chemicals containing functional groups that can promote DNA damage via formation of cross-linkages display significant potency against the bloodstream form of this parasite while exhibiting low toxicity to mammalian cells [14-17]. 
Generated by endogenous metabolic processes and exogenous mutagens, interstrand crosslinks (ICLs) represent a particularly dangerous type of DNA damage. Formed when the complementary strands within the DNA double helix become covalently linked, they block essential cellular processes that require strand separation such as DNA replication and transcription. If left unchecked, they can go on to promote chromosomal fragmentation, rearrangements, or cell death [18-21]. To conserve genome integrity, all cells exploit various combinations of enzymes from "classical" DNA repair pathways to help resolve ICL damage with these constituting an organism's so-called ICL REPAIRtoire. The precise mechanism that repair ICLs are unclear as different systems predominate at different stages in the cell cycle while evolutionarily diverse organisms employ distinct mechanisms to accomplish this task [22-24]. In transcriptionally active mammalian cells, the ICL-mediated stalling of an RNA polymerase complex results in recruitment of factors such as CSB to the lesion site with these subsequently interacting with other DNA repair enzymes including SNM1A, XPG and XPFERCC1 $[25,26]$. In a process known as 'unhooking', the XPF-ERCC1 and XPG endonucleases cleave either side of the crosslinked sugar-phosphate backbone in one of the DNA strands with nucleases, including SNM1A, subsequently degrading the released sequence up to and beyond the ICL [27]. The resultant gap is then filled using damage tolerant translesion synthesis (TLS) DNA polymerases such as $\operatorname{Pol} \zeta, \operatorname{Pol} \eta, \operatorname{Pol} l, \operatorname{Pol} \Theta$ or Pol $\kappa$ with DNA ligase restoring the double strand DNA (dsDNA) structure [28-30]. Once the above has taken place, a second round of nucleotide excision repair (NER), TLS DNA polymerase and DNA ligase activities completely removes the unhooked ICL from the second DNA strand.

In dividing mammalian cells, the ICL-mediated stalling of a single or converging DNA replication fork(s) are recognised by the FANCM helicase. This promotes reversal of the replication fork(s), possibly involving polyubiquitination of PCNA by RAD5-like (e.g. HLTF) activities [31, 32], and recruits a large multi-subunit ubiquitin ligase, termed the Fanconi Anaemia (FA) core complex (FANCA, -B, -C, -E, -F, -G, -L and -T plus the ancillary factors FAAP20, -24 and -100), to the site of the DNA lesion [33-36]. Once formed, this complex monoubiquitinates FANCD2/FANCI with the activated heterodimer drafting a series of nucleases (e.g. FANCQ (XPF)-ERCC1, MUS81-EME1, FAN1 and FANCP (SLX4)) to the site of DNA damage [35, 37]. These cleave the DNA backbone at sequences 5' and 3' to the lesion resulting in the unhooking of the ICL from one of the DNA strands, formation of a single stranded gap and generation of dsDNA breaks (DSBs). The single stranded gap is filled and integrity of the sugar-phosphate backbone is restored by TLS DNA polymerase(s) and DNA ligases activities. This links the parental ICL-containing DNA molecule to one of the newly 
synthesised DNA strands. The ICL is then completely removed by the concerted action of NER, TLS DNA polymerase and DNA ligase activities. The DSB is recognised by the MRN complex (MRE11/RAD50/NBS1) that guide components of the homologous recombination (HR) pathway (e.g. FANCD1 (BRCA2), FANCR (RAD51), FANCO (RAD51C)) to mediate recombination between the broken dsDNA molecule and the newly repaired DNA structure generated from the second round of NER/TLS DNA polymerase/DNA ligase activities. This results in reformation of the Y-shaped forked structure that can serve as a template for recommencement of DNA replication [38]. Due to the bidirectional nature of DNA replication, ICLs can cause the stalling of two converging DNA replication forks [39, 40]. Such X-shaped structures can be resolved using the mechanism outlined above although in this case two of the other newly synthesised DNA strands both form DSBs. In this situation, the above HR mediated repair systems are activated with the newly repaired DNA strand serving as template. The outcome of this event is formation of two dsDNA molecules and the disassociation of the replication machinery.

Little is known about the ICL repair mechanisms expressed by trypanosomes despite these parasites being highly susceptible to ICL-inducing compounds. Some components of the global genome NER (GG-NER) pathway (e.g. TbXPC and TbDDB) may potentially be involved in this process [41] while $T$. brucei cells null for TbSNM1, a member of the SNM1/PSO2 family of nucleases, display sensitivity only to ICL-inducing compounds [42]. Here, using a classical genetics-based approach, we analyse whether other DNA repair enzymes from the parasite's transcription-coupled NER (TC-NER) (TbCSB), HR (TbMRE11, TbRAD51, TbBRCA2 and TbEXO1), mismatch repair (MMR; TbEXO1) and DNA damage tolerance (TbREV3 and TbRAD5) pathways contribute to the trypanosomal ICL repair network then assess their interplay with TbSNM1 and with each other.

\section{Results}

\section{Identifying putative trypanosomal ICL repair enzymes}

Previous studies have shown that MRE11, CSB, EXO1, RAD5 and REV3 all contribute to the yeast and/or mammalian ICL repair systems [25, 26, 43-45]. Using reciprocal BLAST the T. brucei genes encoding for homologues of these enzymes plus their flanking regions were identified from the TriTrypDB (http://tritrypdb.org/tritrypdb/) and NCBI (https://www.ncbi.nlm.nih.gov/) databases. For TbMRE11 and TbCSB, the sequences arising from these searches corresponded to those previously reported [41, 46, 47]. In contrast, the 
genes described here as encoding EXO1, RAD5 and REV3 orthologues have not been characterised. The 2394 bp hypothetical gene (Gene ID: Tb927.4.1480) assigned here as Tbexol has potential to encode for a $86.7 \mathrm{kDa}$ enzyme (TbEXO1) that shares approximately $24 \%$ sequence identity with EXO1/HEX1 exonucleases. The T. brucei homologue contains within its amino terminal the XPG_N (PF00752) and XPG_I (PF00867) domains, that at this position in the protein sequence is a characteristic of this family of enzyme (Fig 1A). A 4839 bp hypothetical open reading frame (Gene ID: Tb927.7.1090), designated here as Tbrad5, was identified as having potential to encode for a homologue of human helicase-like transcription factor (HLTF) and Saccharomyces cerevisiae RAD5. The estimated $179 \mathrm{kDa}$ trypanosomal enzyme (TbRAD5) has approximately $24 \%$ sequence identity to this family of DNA helicase/ubiquitin ligases. Homology is centred on domains responsible for helicase (SNF2_N (PF00176); Helicase_C (PF00271)) and ubiquitin ligase (zf-RING_2 (PF13639)) activities, and DNA binding (HIRAN (PF08797)) (Fig 1B) with the order in which these regions are arranged in the protein backbone being conserved between the parasite, yeast and fungal homologues. A 6181 bp hypothetical gene (Gene ID: Tb927.8.3290; designated as Tbrev3) was postulated to encode for a $217 \mathrm{kDa}$ protein (TbREV3) that has approximately $25 \%$ identity to the error-prone DNA polymerase $\zeta$ catalytic subunit expressed in $S$. cerevisiae and human cells. The parasite enzyme has a domain structure typical of REV3 subunits containing a DNA polymerase family B, exonuclease domain (PF03104) towards its amino terminal and a DNA polymerase family B (PF00136) followed by C4-type zinc finger (PF14260) towards its carboxyl terminal (Fig 1C).

In humans, the FA pathway plays a major role in resolving ICLs encountered during DNA replication. It is now recognised that other eukaryotes also express this system although the activities involved vary between species [48, 49]. To determine the extent of this system in $T$. brucei, FA proteins (complete or specific domains) from humans, Danio rerio, Drosophila melanogaster, Arabidopsis thaliana and S. cerevisiae were used to search for trypanosomal homologues held on the TriTryp and NCBI databases. Out of the 26 sequences examined, $T$. brucei possessed discernible homologues for 7 including BRCA2 (FANCD1), RAD51 (FANCR), RAD51C (FANCO) and XPF (FANCQ), enzymes that play a role in this parasite's HR and NER pathways (Fig 1D) [41, 50-52]. All of the sequences identified were so-called FA effector proteins with none corresponding to components of the FA core complex (FANCA, $\mathrm{B},-\mathrm{C},-\mathrm{E},-\mathrm{F},-\mathrm{G},-\mathrm{L},-\mathrm{M}$ and $-\mathrm{T}$ ), recruitment factors (FANCD2A and -I) or FA associate protein (FAAP10, -16, -20, -24 and -100). 


\section{Construction and validation of $T$. brucei single null mutant lines}

To evaluate whether the identified sequences were involved in the parasite's ICL repair network, cells null for each gene were generated. To achieve this, DNA fragments containing the 5' or 3' flanking or coding sequences from the gene of interest were amplified from wild type T. brucei genomic DNA, digested with restriction enzymes ( 5 ' sequences were processed with SacI and XbaI while 3' sequences were treated with ApaI and KpnI) then cloned either side of a drug resistance cassette that contains the gene encoding for the hygromycin $\mathrm{B}$ phosphotransferase (hyg) or neomycin phosphotransferase (neo) (S1A Fig). The gene deletion/disruption constructs were linearized with SacI/KpnI and the fragments transformed into bloodstream form T. brucei. To remove both allelic copies of the target gene (all sequences tested were single copy genes) two rounds of nucleofection were performed to firstly create heterozygous and then null mutant lines, with clonal populations of the latter tentatively designated as T. brucei $\mathrm{Tb}$ csb $\Delta$, Tbmre11 $\Delta$, Tbexol $\Delta$, Tbrad5 $\Delta$ and Tbrev3 $\Delta$. The predicted effect of each gene disruption event with each integration fragment is depicted (S1B-G Figs).

To confirm that integration of the introduced DNA fragments had occurred into the correct genetic loci, DNA amplification reactions using gDNA as template were performed. Such PCRs used primer combinations that generated fragments specific for the intact targeted gene or each disrupted allele (Fig 2; S1B-G Figs). For example, when using a primer combination designed to detect intact Tbcsb (Tbcsb-KO1/Tbcsb-q2) DNA amplification generated a band of the expected size (approximately $1.4 \mathrm{~kb}$ ) from gDNA extracted from wild type parasites with no band(s) observed in DNA isolated from T. brucei Tbcsb $\Delta$ cells (Fig 2A upper panel; S1B Fig). In contrast, primer combinations that detect the hyg- (Tbcsb-KO1/hyg) or neo(Tbcsb-KO1/neo) interrupted alleles generated amplicons of the predicted size (approximately 1.7 and $1.5 \mathrm{~kb}$, respectively) but only from the gDNA purified from T. brucei $\mathrm{Tb} \operatorname{cs} b \Delta$ null mutant line: No band(s) was observed when using T. brucei wild type gDNA as template.

To show that each $T$. brucei null mutant line was not expressing the targeted gene, DNA amplification reactions were performed on cDNA templates using primer combinations that generated fragments specific for the intact, targeted gene (Fig 2). For example, when using a primer combination designed to generate a Tbcsb specific amplicon (Tbcsb-q1/Tbcsb-q2), a single band of the expected size (approximately $140 \mathrm{bp}$ ) was observed in cDNA synthesised from total RNA extracted from wild type parasites with no band(s) detected in material derived from T. brucei Tbcsb $\Delta$ (Fig 2A lower panel). To confirm that RNA had been extracted from both cell lines and that cDNA had indeed been made, control reactions amplifying Tbtert were 
conducted in parallel. For all tested samples a band of the expected size (approximately 100 bp) was observed.

The above PCR-based strategies were extended to validate T. brucei lines null for Tbmre11, Tbexo1, Tbrad5 or Tbrev3 (Figs 2B-E). In each case, this confirmed that integration of the input DNA fragments had successfully occurred into the parasite genome and established that the deleted transcript was not being expressed in the appropriate null mutant line.

\section{Characterisation of $T$. brucei single null mutant lines}

To assess whether lack of a given DNA repair activity affected parasite growth, the cumulative growth properties of null mutants was determined (S2A Fig). In most cases (Tbexol $\Delta, \mathrm{Tb} c s b \Delta, \mathrm{Tb}$ rad5 $\Delta$ and Tbrev3s), the lines grew at the same rate as wild type with the cultures having a doubling time of around 370 minutes. In contrast, cells lacking TbMRE11 exhibited a slight growth defect, having a mean generation time of 450 minutes, in keeping with previous observations $[46,47]$. To examine these growth characteristics further, the cell cycle progression of Tbmrel1 $\Delta$ and $\mathrm{Tb} \operatorname{cs} b \Delta$ cells was evaluated relative to wild type and Tbsnm1 $\Delta$ lines (S2B Fig). Asynchronous cultures of bloodstream form parasites in the exponential phase of growth were fixed and the ratio of nuclear $(\mathrm{N})$ and mitochondrial (known as the kinetoplast $(\mathrm{K})$ ) genomes within each trypanosome determined with this providing a reliable marker for where that cell is within the cell cycle [53-55]. T. brucei in the G1/S phase of the cell cycle have a $1 \mathrm{~K} 1 \mathrm{~N}$ arrangement, those that possess a $2 \mathrm{~K} 1 \mathrm{~N}$ ratio are said to be in the $\mathrm{G} 2 / \mathrm{M}$ phase while trypanosomes displaying a $2 \mathrm{~K} 2 \mathrm{~N}$ profile are in the post $\mathrm{M}$ phase. For

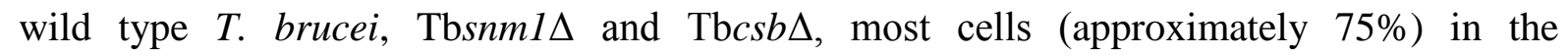
asynchronous population were in G1/S phase, with about $20 \%$ in the G2/M phase and around $5 \%$ in the post $\mathrm{M}$ phase of the cell cycle. In contrast, fewer Tbmre $11 \Delta$ cells (approximately $55 \%$ ) were in the G1/S phase, with a concomitant increase (approximately 40\%) of cells in the G2/M phase of the cell cycle (S5B Fig). Therefore, the increased mean generation time exhibited by TbMRE11-deficient parasites is mostly due to a delay at the G2/M phase of the cell cycle. In other organisms, MRE11 as part of the MRN complex, functions to recognise DSBs generated during DNA replication then aids in recruitment of ATM to the site of DNA damage. Perturbation of this sensor mechanism effects downstream ATM-dependent processes such as progression through the G2/M checkpoint [56].

To evaluate whether deletion of Tbcsb, Tbmre11, Tbexo1, Tbrad5 or Tbrev3 from the $T$. brucei genome altered the parasites sensitivity to DNA damage, the null mutants were grown in the presence of hydroxyurea, phleomycin or methyl methanesulfonate, or cultured following 
249 exposure to a single dose of $\mathrm{UV}$, and the $\mathrm{EC}_{50}$ values towards each treatment determined (Table

250 1; S2C \& D Figs). Cells lacking TbCSB were 5-fold more susceptible to UV than wild type, in 251 keeping with previous observations [41], while parasites lacking TbEXO1 displayed an 252 increased sensitivity (2-fold) towards phleomycin. In keeping with the published literature,

253 TbMRE11 deficient parasites were more susceptible to phleomycin (12-fold) and methyl 254 methanesulfonate (2.5-fold), agents that promote DSBs (Table 1: S2C \& D Figs) [46, 47]. 255 Intriguingly, these mutants were also shown to be 4.9-fold more sensitive to UV, a trait not 256 previously reported, with this phenotype potentially stemming from DSB formation caused by 257 the arrest of replication forks at UV-induced damaged sites [57]. Cells lacking TbRAD5 and 258 TbREV3 were as sensitive to all the DNA damaging treatments tested as wild type indicating 259 that these do not play a front line role in resolving the lesions generated by the tested treatments. 
Table 1. Susceptibility of T. brucei null lines to DNA damaging treatments.

\begin{tabular}{|c|c|c|c|c|}
\hline \multirow[t]{2}{*}{ Treatment } & \multicolumn{4}{|c|}{ T. brucei $\mathrm{EC}_{50}$} \\
\hline & wild type & Tbsnm1A & Tbmre114 & Tbsnm14Tbmre114 \\
\hline Hydroxyurea $(\mu \mathrm{M})$ & $53.37 \pm 3.68$ & $40.62 \pm 2.26(1.3)$ & $43.10 \pm 0.84(1.2)$ & $33.77 \pm 0.38(1.6)$ \\
\hline Phleomycin $(\mu \mathrm{M})$ & $0.12 \pm 0.01$ & $0.12 \pm 0.00(1.0)$ & $0.01 \pm 0.00(12.0)$ & $0.01 \pm 0.00(12.0)$ \\
\hline $\operatorname{MMS}(\mu \mathrm{M})$ & $33.02 \pm 0.28$ & $35.82 \pm 0.29(0.9)$ & $12.63 \pm 0.47(2.5)$ & $15.32 \pm 1.32(2.2)$ \\
\hline \multirow[t]{2}{*}{$\mathrm{UV}(\mathrm{kJ} / \mathrm{cm})$} & $2.58 \pm 0.39$ & $2.66 \pm 0.18(1.0)$ & $0.53 \pm 0.11(4.9)$ & $0.11 \pm 0.09(23.3)$ \\
\hline & wild type & Tbsnm1A & Tbexo14 & Tbsnm14 Tbexo14 \\
\hline Hydroxyurea $(\mu \mathrm{M})$ & $47.56 \pm 1.90$ & $38.62 \pm 2.71(1.2)$ & $52.59 \pm 1.54(0.9)$ & $43.59 \pm 1.84(1.1)$ \\
\hline Phleomycin $(\mu \mathrm{M})$ & $0.12 \pm 0.00$ & $0.12 \pm 0.00(1.0)$ & $0.06 \pm 0.00(2.0)$ & $0.05 \pm 0.00(2.2)$ \\
\hline $\operatorname{MMS}(\mu \mathrm{M})$ & $27.89 \pm 0.63$ & $42.28 \pm 2.89(0.7)$ & $32.46 \pm 0.71(0.9)$ & $40.44 \pm 1.32(0.7)$ \\
\hline \multirow[t]{2}{*}{$\mathrm{UV}(\mathrm{kJ} / \mathrm{cm})$} & $3.54 \pm 0.20$ & $3.90 \pm 0.32(0.9)$ & $2.07 \pm 0.14(1.7)$ & $3.15 \pm 0.98(1.1)$ \\
\hline & wild type & Tbsnm14 & Tbcsb $\Delta$ & Tbsnm1A TbcsbA \\
\hline Hydroxyurea $(\mu \mathrm{M})$ & $71.88 \pm 0.89$ & $67.91 \pm 1.80(1.1)$ & $69.00 \pm 2.67(1.0)$ & $69.77 \pm 2.38(1.0)$ \\
\hline Phleomycin $(\mu \mathrm{M})$ & $0.12 \pm 0.01$ & $0.09 \pm 0.01(1.3)$ & $0.12 \pm 0.01$ & $0.12 \pm 0.01(1.1)$ \\
\hline MMS $(\mu \mathrm{M})$ & $27.78 \pm 5.17$ & $30.94 \pm 13.45(0.9)$ & $26.73 \pm 8.54(1.0)$ & $31.22 \pm 10.4(0.9)$ \\
\hline \multirow[t]{2}{*}{$\mathrm{UV}(\mathrm{kJ} / \mathrm{cm})$} & $2.12 \pm 0.18$ & $2.47 \pm 1.49(0.9)$ & $0.42 \pm 0.14(5.1)$ & $0.47 \pm 0.05(4.5)$ \\
\hline & wild type & Tbsnm1A & Tbrad54 & Tbsnm14 Tbrad5A \\
\hline Hydroxyurea $(\mu \mathrm{M})$ & $67.39 \pm 0.65$ & $50.21 \pm 1.72(1.3)$ & $58.18 \pm 0.39(1.2)$ & $52.20 \pm 1.94(1.3)$ \\
\hline Phleomycin $(\mu \mathrm{M})$ & $0.12 \pm 0.01$ & $0.09 \pm 0.00$ & $0.08 \pm 0.00(1.5)$ & $0.09 \pm 0.00(1.4)$ \\
\hline $\operatorname{MMS}(\mu \mathrm{M})$ & $41.41 \pm 3.56$ & $45.76 \pm 0.65(0.9)$ & $37.82 \pm 1.16(1.1)$ & $45.97 \pm 0.38(0.9)$ \\
\hline \multirow[t]{2}{*}{$\mathrm{UV}(\mathrm{kJ} / \mathrm{cm})$} & $3.61 \pm 0.29$ & $3.17 \pm 0.34(1.1)$ & $3.74 \pm 0.94(1.0)$ & $3.87 \pm 0.35(0.9)$ \\
\hline & wild type & Tbsnm14 & Tbrev3A & Tbsnm14 Tbrev34 \\
\hline Hydroxyurea $(\mu \mathrm{M})$ & $67.39 \pm 0.65$ & $50.21 \pm 1.72(1.3)$ & $50.68 \pm 0.83(1.3)$ & $52.20 \pm 1.76(1.3)$ \\
\hline Phleomycin $(\mu \mathrm{M})$ & $0.12 \pm 0.01$ & $0.09 \pm 0.00$ & $0.09 \pm 0.00$ & $0.08 \pm 0.00$ \\
\hline MMS $(\mu \mathrm{M})$ & $41.41 \pm 3.56$ & $45.76 \pm 0.65(0.9)$ & $38.65 \pm 1.49(1.0)$ & $33.88 \pm 0.70(1.2)$ \\
\hline $\mathrm{UV}(\mathrm{kJ} / \mathrm{cm})$ & $3.46 \pm 0.45$ & $3.92 \pm 1.56(0.9)$ & $3.52 \pm 1.08(1.0)$ & $3.55 \pm 0.73(1.0)$ \\
\hline
\end{tabular}

Data represents $\mathrm{EC}_{50}$ values of various $T$. brucei lines against hydroxyurea (HU), phleomycin, methyl methanesulfonate (MMS) and ultraviolet light (UV). All values are means \pm standard deviations from experiments performed in quadruplicate. The ratio of $\mathrm{EC}_{50}$ values between wild type and single/double null mutant lines is shown as fold difference, in parentheses. 


\section{Susceptibility of $\boldsymbol{T}$. brucei single null mutants towards mechlorethamine}

The above growth inhibition assays were extended to investigate the phenotype displayed by $T$. brucei null mutants towards mechlorethamine, an archetypal ICL inducing agent. The resultant data was plotted as dose response curves from which $\mathrm{EC}_{50}$ values were extrapolated (Fig 3). Previous work has shown that TbSNM1 plays an important role in resolving the damage caused by mechlorethamine with our data confirming this earlier finding [42]: Cells lacking TbSNM1 are >8-fold more susceptible to this compound as compared to controls. A similar alteration in sensitivity was also observed in cells deficient in TbMRE11, TbCSB or TbEXO1 although the difference in $\mathrm{EC}_{50}$ values displayed by these 3 mutants relative to wild type was not as great as that noted for Tbsnm1 $\Delta$ cells; Tbmre $11 \Delta, \operatorname{Tb} c s b \Delta$ and Tbexol $\Delta$ cells were 3-, 4- and 2-fold more susceptible to mechlorethamine than wild type, respectively (Figs 3A-C) This could suggest the relative importance of each enzyme in T. brucei's so-called ICL REPAIRtoire. In contrast, cells lacking TbRAD5 or TbREV3 displayed wild type sensitivities towards the ICL inducing agent indicating they play no significant role in resolving this type of damage (Figs 3D-E). To confirm that the above phenotyping was due to the engineered gene deletion/disruption events and not due to off target effects, the susceptibility of all mutants towards the non-DNA damaging trypanocidal agent difluoromethylornithine was analysed (S3 Fig). In all cases the null lines were as equally sensitive to this compound as each other and the wild type, providing evidence that the above susceptibility profiles are specific for each DNA damaging treatment.

As TbMRE11 functions in T. brucei's HR pathway, the above studies were extended to evaluate whether parasites deficient in other components of this repair system (TbBRCA2 or TbRAD51) exhibited altered susceptibilities towards mechlorethamine: TbBRCA2 and TbRAD51 correspond to two known FA proteins, FANCD1 and FANCR, respectively (Fig 1D). From the resultant dose response curves and $\mathrm{EC}_{50}$ values (Fig S4), parasites lacking TbRAD51 or TbBRCA2 were shown to be 6 - or 3 -fold more sensitive to the ICL inducing agent than controls, respectively, thus implicating these activities in the trypanosomal ICL repair network. The above data implicates TbSNM1, TbCSB, TbEXO1, TbMRE11, TbRAD51 and TbBRCA2 in the bloodstream form T. brucei ICL repair system and thus constitute a part of this parasite's ICL REPAIRtoire.

\section{Mechlorethamine-induced $\gamma \mathrm{H} 2 \mathrm{~A}$ formation in $T$. brucei single null mutants}

Nuclear genome damage in many eukaryotes including $T$. brucei often leads to phosphorylation of $\mathrm{H} 2 \mathrm{~A}$ to form $\gamma \mathrm{H} 2 \mathrm{~A}$ an alteration frequently used to monitor for DNA 
damage including DSB formation $[55,58,59]$. To determine if mechlorethamine can promote this post-translational modification in T. brucei, protein extracts generated from wild type parasites continuously grown in the presence of mechlorethamine $(30,10$ and $3 \mu \mathrm{M})$ were probed for $\gamma \mathrm{H} 2 \mathrm{~A}$ formation using an antiserum against this modified histone (Fig 4A), with the band intensities normalised against untreated controls (Fig 4B): A similar analysis was performed on the same extracts using enolase as loading \& normalisation control. In wild type cells and at all mechlorethamine concentrations tested, an increase in the $\gamma \mathrm{H} 2 \mathrm{~A}$ signal was observed over the first 4 hours of treatment with the marker intensity then declining over the next 4 hours indicating resolution of the DNA damage. When these studies were extended to the null lines, various outcomes were observed. TbSNM1- and TbEXO1-deficient cells behaved similarly to wild type cells. In Tbcsb cells, the formation of $\gamma \mathrm{H} 2 \mathrm{~A}$ was delayed, and continued to increase throughout the experiment suggesting that these parasites may be unable to effectively recognise and resolve any ICL-induced DSBs. Intriguingly, when following $\gamma \mathrm{H} 2 \mathrm{~A}$ formation in cells lacking TbMRE11, no alteration in signal intensity was noted at any time point across any of the mechlorethamine concentrations used indicating that this enzyme plays an important role in phosphorylation of histone H2A in response to DNA damage [60$62]$.

To further evaluate mechlorethamine and DNA damage in mutant lines, cells treated for 4 hours in the presence of the ICL-inducing agent $(30 \mu \mathrm{M})$ were analysed using immunofluorescence microscopy to detect $\gamma \mathrm{H} 2 \mathrm{~A}$ (Fig 4C). For wild type, Tbsnml $\Delta$, Tbcsb $\Delta$ and Tbexol $\Delta$, a discrete signal was observed within the nucleus showing that DNA damage was taking place. This pattern also was detected in wild type, Tbsnml $\Delta$ and Tbexol $\Delta$ cells that had been treated with mechlorethamine for 2 hours but not in $\mathrm{Tb} \operatorname{cs} b \Delta$ parasites. In contrast, and confirming the western blot data, no signal was observed in mechlorethamine treated TbMRE11 null parasites.

\section{Assessing the interplay between trypanosomal ICL repair enzymes}

To evaluate the functional relationship between components of the trypanosomal ICL repair system, the genes encoding for TbEXO1, TbMRE11 or TbCSB were disrupted in a Tbsnm1deficient T. brucei line to create a series of double null mutant lines. Additionally, TbRAD5 and TbREV3 although not primarily involved in resolving ICLs, were also taken forward to determine whether their activities in this repair network becomes apparent in the absence of TbSNM1: The role of some yeast factors in ICL repair are only apparent when the activity of another DNA repair protein is missing [63]. To generate the double null mutant lines, the hyg- 
and neo-based integration vectors for each targeted gene were linearised and sequentially transformed into T. brucei Tbsnml $1 \Delta$ cells (in this mutant line, Tbsnml has been disrupted using vectors based around resistance cassettes that includes the gene encoding for blasticidin-Sdeaminase (bla) or puromycin $\mathrm{N}$-acetyltransferase (pac) [42]). Following selection, all putative double null clones were validated using the PCR-based strategies outlined previously with this confirming that the introduced DNA fragments had integrated into the T. brucei genome and that the recombinant parasites were no longer expressing both disrupted genes (Fig 2).

Analysis of the double null mutants revealed that parasites lacking TbSNM1 and TbCSB, TbEXO1, TbRAD5 or TbREV3 grew roughly at the same rate as wild type while cells deficient in TbSNM1 and TbMRE11 exhibited a slightly longer mean generation time comparable to that noted for the T. brucei Tbmre11 line. Additionally, phenotypic screening with DNA damaging treatments demonstrated that the susceptibility displayed by the double null mutant lines were generally equivalent to the more sensitive phenotype shown by the single null for each gene pairing (Table 1). For example, all Tbcsb-deficient cell types were approximately 5fold more susceptible to UV than wild type or Tbsnm1 $\Delta$ such that the Tbsnml $1 \mathrm{~Tb} \operatorname{cs} b \Delta$ line exhibited an $\mathrm{EC}_{50}$ similar to Tbcsb $\Delta$ parasites. As such, lack of TbSNM1 and TbCSB activities in the same cell does not lead to an increase in susceptibility to UV. The only situation where a combinatorial effect on susceptibility was noted related to cells lacking TbSNM1 and TbMRE11. Intriguingly, such double null mutant parasites were more than 20-fold more sensitive to UV-induced lesions relative to T. brucei wild type and Tbsnm1 $\Delta$, and 4-fold more sensitive relative to Tbmre 11 (Table 1). This difference could reflect that TbSNM1 does play a secondary role in resolving UV-induced damage, a phenotype that is only apparent in cells compromised in the HR pathway.

The phenotypic screens were extended to investigate the susceptibility that T. brucei double null mutants display towards mechlorethamine with the resultant data plotted as dose response curves from which $\mathrm{EC}_{50}$ values were extrapolated (Fig 3). This revealed that cells lacking TbSNM1 and TbMRE11 were hypersensitive towards this ICL-inducing agent relative to wild type and the Tbsnm1 $\Delta$ or Tbmre11 1 lines showing that these two nucleases function in a nonepistatic fashion and do not operate in the same ICL repair system (Fig 3C). In contrast, parasites deficient in TbSNM1 and TbCSB or in TbSNM1 and TbEXO1 exhibit dose response sensitivities to mechlorethamine similar to Tbsnml $\Delta$ parasites, with the double null lines being more susceptible to this compound than wild type and Tbcsb $\Delta$ or Tbexol $\Delta$ cells (Figs $3 \mathrm{~A} \&$ B). The type of patterns observed indicate that TbSNM1 functions epistatically with TbCSB and TbEXO1. When the susceptibility phenotype of trypanosomes lacking TbSNM1 and 
TbRAD5 or TbREV3 towards mechlorethamine was assessed, dose response curves similar to that obtained using control lines were observed (Figs 3D \& E). This suggests that TbRAD5 and TbREV3 do not play a role in the T. brucei ICL repair networks.

To further investigate any epistatic/non-epistatic relationships, double null lines lacking TbCSB and TbMRE11, TbEXO1 and TbMRE11 or TbCSB and TbEXO1 activities were made and the resultant lines validated (Figs 5A-C). Evaluation of dose response curves of parasites lacking TbCSB and TbEXO1 towards mechlorethamine revealed that these cells exhibited a susceptibility pattern similar to $\mathrm{Tb} c s b \Delta$ trypanosomes, with the double null lines being more sensitive to this treatment than wild type and Tbexol $\Delta$ cells (Fig 5D). This clearly demonstrates that these Tbcsb is epistatic with respect to Tbexo 1 in the T. brucei ICL repair system. In contrast, Tbcsb $\Delta$ Tbmre $11 \Delta$ and Tbexol $\Delta$ Tbmre $11 \Delta$ trypanosomes were hypersensitive to this ICL-inducing agent relative to wild type cells and the corresponding single null mutant lines providing evidence that TbCSB and TbEXO1 both function in a non-epistatic fashion with respect to TbMRE11 and as such the former two activities operate in an ICL repair system distinct from that the one the latter is involved in (Figs 5E and F).

All of the double null mutant data clearly shows that $T$. brucei expresses at least two distinct ICL repair systems with one involving the concerted action of TbSNM1, TbCSB and TbEXO1 with the other using TbMRE11. It is tempting to speculate that given TbCSB plays a role in TC-NER and that the former SNM1-dependent pathway functions to resolve ICLs encountered during DNA transcription while the MRE11-dependent system helps remove ICLs encountered during DNA replication.

\section{Discussion}

Throughout its cell and life cycle $T$. brucei may be exposed to a range of endogenous metabolites and environmental stimuli that in other organisms result in ICL formation [64-66]. Despite ICLs being highly toxic, very little is known about the mechanisms T. brucei employs to resolve such lesions. The ICL repair networks expressed in yeast and mammalian cells can be distinguished by how these systems are activated and the factors involved [18, 21, 22, 24, 67]. Throughout the cell cycle, but most prominently during the G1/G0-phases, replicationindependent systems operate with these mechanisms triggered by global genome surveillance processes (by GG-NER) or stalling of transcription complexes (by TC-NER) [25, 26, 68]. Following lesion detection, networks are invoked that involve components from the NER and/or MMR pathways which together unhook then resect the ICL from the genome with any excised genetic material replaced by TLS $[28,69]$. In contrast, during the S-phase of the cell 
cycle, replication-dependent mechanism(s) function to eliminate ICLs [31, 35-37, 63, 70]. In such networks, recognition of an ICL stalled DNA replication fork(s) occurs through components of the FA or Fanconi-like systems that firstly cause reversal of the replication fork(s) then guide predominantly HR effector proteins to the site of damage. In conjunction with the endonucleases centred on XPF-ERCC1 activity, these cooperate to remove and replace the cross linkage from the genome using homologous sequences as a repair template. Here, using comparative genomics in conjunction with classical genetic approaches we have identified several putative trypanosomal ICL repair proteins and established whether the in silico assignments were of biological relevance. This revealed that enzymes from the T. brucei NER, HR and/or MMR pathways contribute to this organism's ICL REPAIRtoire to resolve damage generated by mechlorethamine and that these activities function across two distinct networks.

An informatics approach was employed to determine the extent of the T. brucei ICL REPAIRtoire. Using protein sequences from representative members of the yeast and/or mammalian ICL repair systems as bait, genome database searches were performed identifying trypanosomal homologues of the TC-NER helicase CSB, the MMR/HR exonuclease EXO1 and the REV3 catalytic subunit of pol $\zeta$ involved in error-prone TLS were identified. Additionally, the ICL repair specific nuclease TbSNM1, several HR components including MRE11, RAD51 and BRCA2, and RAD5, a DNA helicase/ubiquitin ligase that can promote HR in a template switching, error-free DNA damage tolerance pathway, were found. Intriguingly, no discernible homologues for any component of the FA core complex (FANCA, $-\mathrm{B},-\mathrm{C},-\mathrm{E},-\mathrm{F},-\mathrm{G},-\mathrm{L}$ and $-\mathrm{T}$ plus the ancillary factors FAAP20, -24 and -100), recruitment factors (FANCD2A and $-\mathrm{I}$ ) or FA-like proteins (Mph1 and Slx4) were identified suggesting the ICL-mediated stalling of DNA replication forks is recognised by trypanosomal mechanisms distinct from those typically expressed by other eukaryotes with this potentially triggering unusual downstream responses.

In many cases, the use of null mutant lines has provided the primary route for the identification of which DNA repair factors function in an organism's ICL REPAIRtoire and in deciphering how these components interact $[45,63,71,72]$. Transferring this approach to $T$. brucei, lines lacking a single potential ICL repair factor were made (TbCSB, TbEXO1, TbMRE11, TbRAD5 or TbREV3) or sourced (TbSNM1, TbRAD51 or TbBRCA2 [42, 50, 52]) with these subsequently phenotyped using mechlorethamine as a selective agent. For most parasite lines tested, an increased susceptibility to the ICL-inducing agent was observed, demonstrating that TbSNM1, TbCSB, TbEXO1, TbMRE11, TbRAD51 and TbBRCA2 all 
contribute to the trypanosomal ICL REPAIRtoire. Comparison of the degree of susceptibility of each mutant in relation to controls suggests that TbSNM1 may play a prominent role in resolving crosslinks while TbCSB, TbMRE11, TbRAD51 and TbBRCA2 all represent important factors in these repair systems: Tbsnm1 $\triangle$ parasites exhibited the highest fold (around 7.5-fold) difference in susceptibility while Tbcsb $\Delta$, Tbmre11 14 , Tbrad51 $\Delta$ and Tbbrac2 $\Delta$ cells displayed moderate (between 3- to 5-fold) changes. In the case of TbEXO1, cells lacking this exonuclease displayed about a 2-fold increase in sensitivity indicting that this enzyme may play an ancillary role in the trypanosomal ICL repair network. When these screens were extended to TbRAD5- or TbREV3-deficient parasites no obvious differences in susceptibility was observed. As with other organisms, trypanosomes express several error-free and error-prone DNA damage tolerance mechanisms and it is plausible that these other systems may complement for the lack of TbRAD5 or TbREV3 activity in the corresponding T. brucei null line [73].

Resolution of ICLs involves the concerted action of various enzymes from different 'classical' DNA repair pathways operating across several cell cycle dependent networks and all within a single cell. In yeast, the interplay of ICL repair factors and their assignment to a given pathway frequently involves use of fungal lines lacking multiple DNA repair activities and determining their susceptibility towards an ICL-inducing agent [45, 63, 71, 72]. Such studies can then help inform the situation in other organisms. To evaluate the interactions of TbSNM1, TbCSB, TbEXO1 and TbMRE11, T. brucei lines lacking various combinations of two of these activities were generated with the parasites subsequently phenotyped against mechlorethamine. This revealed that TbSNM1, TbCSB and TbEXO1 function in one T. brucei ICL repair system with TbMRE11 operating as part of a second distinct network. This situation resembles that noted in other organisms although there are some key differences. In $S$. cerevisiae, PSO2 (the yeast SNM1 homologue) displays a non-epistatic interaction with EXO1 and all tested DSB repair protein, including MRE11 [45, 72, 74, 75]. It also associates with several NER factors such as RAD1 (XPF), RAD3 (XPD), RAD4 (XPC) and RAD14 (XPA) $[22,72]$ although whether it interacts with RAD26 (the yeast CSB homologue) remains unclear [22]. In humans, the role played by SNM1 is reportedly more complex partly because each cell can express multiple isoforms of this family of nuclease [76]. In the case of SNM1A, the human homologue most like yeast PSO2, a direct interaction with CSB has been established [26] with associations also noted for other NER factors such as XPC, DDB2 and XPF-ERCC1 [27, 68]. In a situation contrary to that observed in yeast and T. brucei, SNM1A also appears to play a role in replication-dependent ICL resolving mechanisms as it reportedly co-localises with the 
HR factors such as MRE11 and BRCA1 while human cells with depleted levels for this activity exhibit an increase in replication associated DSBs [27, 77]. Additionally, human cells also express a second SNM1/PSO2 isoform called SNM1B that has been shown to physically interact with MRE11 via an MRN binding site with this activity facilitating direct DNA repair at the site of stalled DNA replication forks, a HR-dependent process that occurs during S-phase of the cell cycle $[76,78,79]$. Therefore, it appears that in single-celled eukaryotes members of the SNM1/PSO2 family function only in replication-independent ICL repair systems while in higher eukaryotes this class of nuclease additionally play roles in replication-dependent ICL repair processes.

Based on the pathways reported in other organisms coupled with observations made by ourselves and by Machado et al [41], we postulate that in bloodstream form T. brucei ICLs encountered throughout the cell cycle as a result of global genome surveillance through GGNER (e.g. TbXPC, TbDDB, TbRAD23) or transcription complex stalling by TC-NER (e.g. TbCSB) recognition mechanisms trigger TbXPF-TbERCC1 (and possibly XPG) incision of one DNA strand at sites flanking the ICL (Fig 6A). The resultant unhooked sequence subsequently undergoes nucleolytic processing, a process carried out by TbSNM1 (and possibly TbEXO1), up to and beyond the crosslink, leaving a single nucleotide tethered to the complementary strand. Replication Protein A binds to the single stranded gap generated by the unhooking activity that via activated PCNA recruits TLS DNA polymerases to this site. These in conjunction with DNA ligases restore the integrity of the DNA strand. To completely remove the crosslinked nucleotide from the other DNA strand a second round of incision followed by TLS activity occurs to generate the undamaged dsDNA structure. In contrast and representing a DNA replication-dependent mechanism, two replication forks converge upon an ICL to form an X-shaped structure (Fig 6B). Following recognition by an unknown mechanism that does not involve the FA core or FA-like proteins, incision of one DNA strand at sites flanking the ICL occurs possibly through the action of TbXPF-TbERCC1, TbMUS18 and/or TbFAN1. This results in unhooking of the ICL from one DNA strand, formation of a single stranded gap and generation of DSBs, with the latter detected through the TbMRE11-mediated formation of $\gamma \mathrm{H} 2 \mathrm{~A}$. The single stranded gap can be filled as described above through TLS DNA polymerase/DNA ligase activities with the unhooked sequence removed and replaced by a second round of incision and TLS: The unhooked sequence may undergo nucleolytic processing prior to the $2^{\text {nd }}$ incision event in an SNM1-independent mechanism possibly involving TbEXO1. The DSBs can then be repaired by HR involving TbRAD51 and TbBRCA2, using the newly formed dsDNA as a template. The two proposed trypanosomal 
pathways both employ TLS activities to fill single strand gaps that are associated with unhooking events. However certain DNA polymerases such as pol $\zeta$ are error prone and as such their activity may introduce base mismatches in the newly 'repaired' sequence. To overcome such secondary lesions, MMR mechanisms possibly involving TbEXO1 can operate although these may create point mutations relative to the initial sequence.

We have now shown that $T$. brucei expresses several enzymes from a number of classical DNA repair pathways that also function to resolve ICLs. As in other organisms, these activities operate across multiple, distinct pathways that we postulate function to fix lesions encountered at different points in the cell cycle. The comparative analysis performed here on a relatively small number of targets has already shown that the ICL resolving mechanisms employed by $T$. brucei share similarities to those expressed by other eukaryotes but has also highlighted some intriguing differences. By further unravelling the ICL repair systems additional variations may be identified. Such disparities between the trypanosomal and mammalian networks could identify potential targets that may be exploitable in terms of drug development such that the specific inhibition of a parasite ICL repair factor(s) may render the pathogen more susceptible to compounds that promote ICL formation.

\section{Material and methods}

\section{Parasite culture}

Bloodstream form Trypanosoma brucei brucei MITat 427 (clone 221a) (designated as wild type) was grown in HMI-9 (Invitrogen) medium supplemented with $3 \mathrm{~g}^{-1}$ sodium bicarbonate, $0.014 \%(\mathrm{v} / \mathrm{v}) \beta$-mercaptoethanol and $10 \%(\mathrm{v} / \mathrm{v})$ foetal bovine serum (FBS) at $37{ }^{\circ} \mathrm{C}$ under a $5 \%(\mathrm{v} / \mathrm{v}) \mathrm{CO}_{2}$ atmosphere [80]. Derivatives of the '221' line null for DNA repair enzymes (S1 Table), including cells lacking Tbsnm1, Tbrad51 and Tbbrca2 [42, 50, 52], were maintained in the above growth medium containing $2.5 \mu \mathrm{g} \mathrm{ml}^{-1}$ hygromycin, $10 \mu \mathrm{g} \mathrm{ml}^{-1}$ blasticidin, $2 \mu \mathrm{g}$ $\mathrm{ml}^{-1}$ puromycin and/or $2 \mu \mathrm{g} \mathrm{ml} \mathrm{l}^{-1} \mathrm{G} 418$.

\section{Chemicals \& treatments}

Agents that promote DNA damage were obtained from Sigma-Aldrich (methyl methanesulfonate, hydroxyurea, phleomycin) or Cambridge Biosciences (mechlorethamine) while UV irradiation was performed with a Stratalinker UV crosslinker (Stratagene). The trypanocidal selective compounds blasticidin, puromycin, G418 and hygromycin were sourced 
from Melford Laboratories Ltd with difluoromethylornithine supplied by Prof. Mike Barrett (University of Glasgow).

\section{Nucleic acids}

The Tbcsb (Tb927.7.4080), Tbmre11 (Tb927.2.4390), Tbexol (Tb927.8.3220), Tbrad5 (Tb927.7.1090) and Tbrev3 (Tb927.8.3290) open reading frames and flanking regions were identified following reciprocal BLAST searches of TriTrypDB (http://tritrypdb.org/tritrypdb/) and NCBI database (https://www.ncbi.nlm.nih.gov/) using the human and/or yeast orthologue as bait, and analysis of trypanosomal sequence information in the published literature [41, 46, 47, 81]. Deduced protein sequences were aligned to the counterparts expressed by other organisms using CLUSTAL $\Omega$ (http://www.ebi.ac.uk/Tools/msa/clustalo/), domain structures evaluated using HMMR (http://www.ebi.ac.uk/Tools/hmmer/) and regions of homology highlighted using BoxShade (https://embnet.vital-it.ch/software/BOX_form.html).

\section{Construction of null mutant lines}

The vectors used to delete or interrupt a target gene in the T. brucei genome were generated as follows. For Tbcsb, sequences corresponding to 5' and 3' flanking regions were amplified from $T$. brucei genomic DNA and sequentially cloned either side of a resistance cassette that includes the gene encoding for hygromycin $\mathrm{B}$ phosphotransferase or neomycin phosphotransferase. The vectors designed to disrupt Tbexo1, Tbmre 11, Tbrad5 or Tbrev3 were made in a similar way except that the amplified fragments were derived from the 5' and 3' ends of the coding sequence and these were cloned either side of a resistance cassette that included the gene encoding for hyg, neo, bla or pac: The primers and primer combinations used to generate the 5' and 3' sequences and the sizes of the amplified fragments are shown in S2 and S3 Tables, and S1A Fig. Constructs were linearized (SacI/KpnI for the hyg, neo and pac constructs and SacII/ KpnI for the bla vector) then transformed into bloodstream form T. brucei using the Human T-cell nucleofection ${ }^{\circledR}$ kit and an Amaxa ${ }^{\circledR}$ Nucleofector ${ }^{\mathrm{TM}}$ (Lonza AG) set to program X-001. As the T. brucei genes targeted here are single copy in a diploid genome, two rounds of nucleofection were needed to firstly create heterozygous and then null mutant lines. Integration of the Tbcsb-based DNA constructs into the T. brucei genome resulted in deletion of the entire open reading frame. In contrast, integration of the Tbexo1-, Tbmre11-, Tbrad5- or Tbrev3-based gene disruption constructs into the T. brucei genome resulted in deletion of 50, 37,67 or $80 \%$ of the corresponding open reading frame, respectively. The regions removed from the genome sequences are postulated to be needed for DNA binding (TbEXO1, 
TbMRE11, TbRAD5), nuclease (TbEXO1, TbMRE11, TbREV3), ubiquitin ligase (TbRAD5) or DNA polymerase (TbREV3) activities.

\section{Validation of recombinant parasites}

To demonstrate that integration of a gene interruption cassette had occurred at the correct genetic loci and confirm that the T. brucei null mutant line was no longer expressing the targeted gene(s), DNA amplification reactions were performed on parasite genomic DNA or cDNA templates. For gene interruption constructs, the primer combinations used generated amplicons specific for the intact targeted gene or the hyg-, neo-, pac- and/or bla-disrupted allele. For all DNA's analysed, additional reactions aimed at detecting Tbtert were performed on cDNA to check the integrity of these templates and as loading control. The primer sequences and combinations used in cell line validation are listed in S2 and S3 Tables.

\section{Growth curves}

Bloodstream form T. brucei in the logarithmic phase of growth were seeded at $1 \times 10^{4}$ parasites $\mathrm{ml}^{-1}$ in parasite growth medium and incubated at $37{ }^{\circ} \mathrm{C}$ under a $5 \%(\mathrm{v} / \mathrm{v}) \mathrm{CO}_{2}$ atmosphere. Each day, the cell density of each culture was measured using a Neubauer haemocytometer. When the number of parasites reached approximately $1 \times 10^{6} \mathrm{ml}^{-1}$, a new culture seeded at $1 \times 10^{4}$ parasites $\mathrm{ml}^{-1}$ was set up. This analysis was carried out over an 8 to 14 day period. Growth curves were generated using GraphPad Prism (GraphPad Software Inc.). All growth assays were performed in triplicate and each count at each time point expressed as a mean \pm standard deviation. Mean generation times were calculated using http://www.doubling-time.com/compute.php.

\section{Microscopy and protein analysis}

Bloodstream form T. brucei were fixed in growth medium with an equal volume of $2 \%$ $(\mathrm{w} / \mathrm{v})$ paraformaldehyde/PBS, washed once in phosphate buffered saline (PBS) and aliquots containing approximately $10^{5}$ cells air dried onto a microscope slide. For cell cycle arrest assays, the genomes of paraformaldehyde-fixed trypanosomes were stained with 4',6diamidino-2-phenylindole (DAPI) and trypanosomes visualized using a Leica DMRA2 epifluorescent microscope in conjunction with a C4742-95 digital camera (Hamamatsu Photonics). For immunofluorescence studies, paraformaldehyde-fixed trypanosomes were permeabilized for 15 minutes in $0.5 \%$ (v/v) Triton-X (Sigma-Aldrich) in PBS, blocked with $50 \%$ (v/v) FBS in PBS then labelled with rabbit anti- $\gamma \mathrm{H} 2 \mathrm{~A}$ (Dr Richard McCulloch, University 
of Glasgow) antisera diluted 1:250 in $3 \%(\mathrm{v} / \mathrm{v})$ FBS in PBS. After 45 minutes, the slides were washed extensively in PBS then incubated for 45 minutes with Alexa-Fluor 488 goat antimouse (Molecular Probes) diluted 1:1000 in $3 \%$ (v/v) FBS in PBS. Following further PBS washes, the parasite DNA was stained using Vectashield Mounting Medium containing DAPI (Vectorshield Laboratories). Images were captured using a Leica SP5 confocal microscope (Leica Microsystems (UK) Ltd). All images were processed and corrected fluorescence intensities determined using ImageJ.

For western blot analysis, protein extracts from approximately $2 \times 10^{6}$ trypanosomes were probed with rabbit anti- $\gamma \mathrm{H} 2 \mathrm{~A}$ and rabbit anti-enolase (Prof Paul Michels, University of Edinburgh) antisera used at 1:1000 or 1:150,000 dilution, respectively, followed by goat antirabbit IRDye ${ }^{\mathrm{TM}} 800 \mathrm{CW}$ antibody (LI-COR) diluted at 1:5,000. Detection of the near infrared signal was monitored using an Odyssey ${ }^{\circledR} \mathrm{CLx}$ infrared imaging system (LI-COR). Band signal intensity measurements were carried out using the Analysis Tool in Image Studio ${ }^{\mathrm{TM}}$ Lite (LiCOR Biosciences).

\section{Susceptibility screens}

All growth inhibition assays were carried out in a 96-well plate format (ThermoFisher Scientific). Bloodstream form T. bruce i in the logarithmic phase of growth were seeded at $1 \mathrm{x}$ $10^{4} \mathrm{ml}^{-1}\left(1 \times 10^{5} \mathrm{ml}^{-1}\right.$ for Tbrad51 4 and Tbbrca2 4$)$ in $200 \mu 1$ growth medium containing different concentrations of the compound under study: For UV irradiation, parasites received doses up to $9000 \mathrm{~J} \mathrm{~m}^{-2}$ using a Stratalinker ${ }^{\circledR} \mathrm{UV}$ crosslinker (Stratagene). After incubation at $37{ }^{\circ} \mathrm{C}$ under a $5 \% \mathrm{CO}_{2}$ atmosphere for 3 days, resazurin (Sigma Aldrich) was added to each well at a final concentration of $12.5 \mu \mathrm{g} \mathrm{ml}^{-1}$ (or $2.5 \mu \mathrm{g}$ per well). The plates were further incubated at $37{ }^{\circ} \mathrm{C}$ under a $5 \% \mathrm{CO}_{2}$ for 6 to 8 hours before measuring the fluorescence of each culture using a Gemini Fluorescent Plate reader (Molecular Devices) set at $\lambda_{\mathrm{EX}}=530 \mathrm{~nm}$ and $\lambda_{\mathrm{EM}}=585 \mathrm{~nm}$ with a filter cut off at $550 \mathrm{~nm}$. The change in fluorescence resulting from the reduction of resazurin is proportional to the number of live cells. A drug/treatment concentration that inhibits cell growth by $50 \%\left(\mathrm{EC}_{50}\right)$ was established using the non-linear regression tool on GraphPad Prism (GraphPad Software Inc.).

\section{Acknowledgement}

We thank Prof Paul Michels (University of Edinburgh) for the T. brucei enolase antibody, Dr Richard McCulloch (University of Glasgow) for the anti-T. brucei $\gamma \mathrm{H} 2 \mathrm{~A}$ antisera and the 
T. brucei Tbrad51 and Tbbrca2 null mutant lines, and Heidi Bowring, Charlotte Chaloner, Mariam Kassir and Shiji Zhao (Queen Mary University of London BSc or MSc students) who undertook their research projects in eth Wilkinson lab and contributed to making some of the DNA vectors/cell lines used in this study. We would like to thank Dr Peter Thorpe (Queen Mary University of London) and Dr Martin Taylor (London School of Hygiene \& Tropical Medicine) for valuable discussions and comments on the manuscript. Ambika Dittani was funded by Queen Mary University of London College Studentship.

\section{References}

1. Buscher P, Cecchi G, Jamonneau V, Priotto G. Human African trypanosomiasis. Lancet. 2017;390(10110):2397-409.

2. Steverding D. The history of African trypanosomiasis. Parasit Vectors. 2008;1(1):3.

3. Franco JR, Simarro PP, Diarra A, Ruiz-Postigo JA, Jannin JG. The journey towards elimination of gambiense human African trypanosomiasis: not far, nor easy. Parasitology. 2014;141(6):748-60.

4. Franco JR, Cecchi G, Priotto G, Paone M, Diarra A, Grout L, et al. Monitoring the elimination of human African trypanosomiasis: Update to 2014. PLoS Negl Trop Dis. 2017;11(5):e0005585.

5. Wilkinson SR, Kelly JM. Trypanocidal drugs: mechanisms, resistance and new targets. Expert Rev Mol Med. 2009;11:e31.

6. Babokhov P, Sanyaolu AO, Oyibo WA, Fagbenro-Beyioku AF, Iriemenam NC. A current analysis of chemotherapy strategies for the treatment of human African trypanosomiasis. Pathog Glob Health. 2013;107(5):242-52.

7. Wilkinson SR, Taylor MC, Horn D, Kelly JM, Cheeseman I. A mechanism for crossresistance to nifurtimox and benznidazole in trypanosomes. Proc Natl Acad Sci U S A. 2008;105(13):5022-7.

8. Vincent IM, Creek D, Watson DG, Kamleh MA, Woods DJ, Wong PE, et al. A molecular mechanism for eflornithine resistance in African trypanosomes. PLoS Pathog. 2010;6(11):e1001204.

9. Alsford S, Eckert S, Baker N, Glover L, Sanchez-Flores A, Leung KF, et al. High-throughput decoding of antitrypanosomal drug efficacy and resistance. Nature. 2012;482(7384):232-6.

10. Baker N, de Koning HP, Maser P, Horn D. Drug resistance in African trypanosomiasis: the melarsoprol and pentamidine story. Trends Parasitol. 2013;29(3):110-8.

11. Wyllie S, Foth BJ, Kelner A, Sokolova AY, Berriman M, Fairlamb AH. Nitroheterocyclic drug resistance mechanisms in Trypanosoma brucei. J Antimicrob Chemother. 2016;71(3):625-34.

12. Fairlamb AH, Horn D. Melarsoprol Resistance in African Trypanosomiasis. Trends Parasitol. 2018;34(6):481-92.

13. Hall BS, Bot C, Wilkinson SR. Nifurtimox activation by trypanosomal type I nitroreductases generates cytotoxic nitrile metabolites. J Biol Chem. 2011;286(15):13088-95.

14. Bot C, Hall BS, Bashir N, Taylor MC, Helsby NA, Wilkinson SR. Trypanocidal activity of aziridinyl nitrobenzamide prodrugs. Antimicrob Agents Chemother. 2010;54(10):4246-52. 
15. Hall BS, Wu X, Hu L, Wilkinson SR. Exploiting the drug-activating properties of a novel trypanosomal nitroreductase. Antimicrob Agents Chemother. 2010;54(3):1193-9.

16. Sokolova AY, Wyllie S, Patterson S, Oza SL, Read KD, Fairlamb AH. Cross-resistance to nitro drugs and implications for treatment of human African trypanosomiasis. Antimicrob Agents Chemother. 2010;54(7):2893-900.

17. Hu LQ, Wu XH, Han JY, Chen L, Vass SO, Browne P, et al. Synthesis and structureactivity relationships of nitrobenzyl phosphoramide mustards as nitroreductaseactivated prodrugs. Bioorg Med Chem Lett. 2011;21(13):3986-91.

18. Dronkert ML, Kanaar R. Repair of DNA interstrand cross-links. Mutat Res. 2001;486(4):217-47.

19. McHugh PJ, Spanswick VJ, Hartley JA. Repair of DNA interstrand crosslinks: molecular mechanisms and clinical relevance. Lancet Oncol. 2001;2(8):483-90.

20. Deans AJ, West SC. DNA interstrand crosslink repair and cancer. Nature Rev Cancer. 2011;11(7):467-80.

21. Sengerova B, Wang AT, McHugh PJ. Orchestrating the nucleases involved in DNA interstrand cross-link (ICL) repair. Cell Cycle. 2011;10(23):3999-4008.

22. Lehoczky $\mathrm{P}$, McHugh PJ, Chovanec M. DNA interstrand cross-link repair in Saccharomyces cerevisiae. FEMS Microbiol Rev. 2007;31(2):109-33.

23. McVey M. Strategies for DNA interstrand crosslink repair: insights from worms, flies, frogs, and slime molds. Environ Mol Mutagen. 2010;51(6):646-58.

24. Hashimoto S, Anai H, Hanada K. Mechanisms of interstrand DNA crosslink repair and human disorders. Genes Environ. 2016;38:9.

25. Enoiu M, Jiricny J, Scharer OD. Repair of cisplatin-induced DNA interstrand crosslinks by a replication-independent pathway involving transcription-coupled repair and translesion synthesis. Nucleic Acids Res. 2012;40(18):8953-64.

26. Iyama T, Lee SY, Berquist BR, Gileadi O, Bohr VA, Seidman MM, et al. CSB interacts with SNM1A and promotes DNA interstrand crosslink processing. Nucleic Acids Res. 2015;43(1):247-58.

27. Wang AT, Sengerova B, Cattell E, Inagawa T, Hartley JM, Kiakos K, et al. Human SNM1A and XPF-ERCC1 collaborate to initiate DNA interstrand cross-link repair. Genes Dev. 2011;25(17):1859-70.

28. Sarkar S, Davies AA, Ulrich HD, McHugh PJ. DNA interstrand crosslink repair during G1 involves nucleotide excision repair and DNA polymerase zeta. EMBO J. 2006;25(6):1285-94.

29. Ho TV, Guainazzi A, Derkunt SB, Enoiu M, Scharer OD. Structure-dependent bypass of DNA interstrand crosslinks by translesion synthesis polymerases. Nucleic Acids Res. 2011;39(17):7455-64.

30. Sharma S, Canman CE. REV1 and DNA polymerase zeta in DNA interstrand crosslink repair. Environ Mol Mutagen. 2012;53(9):725-40.

31. Daee DL, Ferrari E, Longerich S, Zheng XF, Xue X, Branzei D, et al. Rad5-dependent DNA repair functions of the Saccharomyces cerevisiae FANCM protein homolog Mph1. J Biol Chem. 2012;287(32):26563-75.

32. Quinet A, Lemacon D, Vindigni A. Replication Fork Reversal: Players and Guardians. Mol Cell. 2017;68(5):830-3.

33. Gari K, Decaillet C, Stasiak AZ, Stasiak A, Constantinou A. The Fanconi anemia protein FANCM can promote branch migration of Holliday junctions and replication forks. Mol Cell. 2008;29(1):141-8.

34. Gari K, Decaillet C, Delannoy M, Wu L, Constantinou A. Remodeling of DNA replication structures by the branch point translocase FANCM. Proc Natl Acad Sci U S A. 2008;105(42):16107-12. 
35. Moldovan GL, D'Andrea AD. How the fanconi anemia pathway guards the genome. Annu Rev Genet. 2009;43:223-49.

36. Tan W, Deans AJ. A defined role for multiple Fanconi anemia gene products in DNAdamage-associated ubiquitination. Exp Hematol. 2017;50:27-32.

37. Ceccaldi R, Sarangi P, D'Andrea AD. The Fanconi anaemia pathway: new players and new functions. Nat Rev Mol Cell Biol. 2016;17(6):337-49.

38. Wang W. Emergence of a DNA-damage response network consisting of Fanconi anaemia and BRCA proteins. Nat Rev Genet. 2007;8(10):735-48.

39. Chaudhury I, Stroik DR, Sobeck A. FANCD2-controlled chromatin access of the Fanconiassociated nuclease FAN1 is crucial for the recovery of stalled replication forks. Mol Cell Biol. 2014;34(21):3939-54.

40. Zhang J, Walter JC. Mechanism and regulation of incisions during DNA interstrand crosslink repair. DNA Repair (Amst). 2014;19:135-42.

41. Machado CR, Vieira-da-Rocha JP, Mendes IC, Rajao MA, Marcello L, Bitar M, et al. Nucleotide excision repair in Trypanosoma brucei: specialization of transcriptioncoupled repair due to multigenic transcription. Mol Microbiol. 2014;92(4):756-76.

42. Sullivan JA, Tong JL, Wong M, Kumar A, Sarkar H, Ali S, et al. Unravelling the role of SNM1 in the DNA repair system of Trypanosoma brucei. Mol Microbiol. 2015;96(4):827-38.

43. Siedel W, Brendel M. Mutant Gene snm2-1 (ts), conferring thermoconditional mutagen sensitivity in Saccharomyces cerevisiae, is allelic with RAD5. Curr Genet. 1982;5(2):93-5.

44. Cassier-Chauvat C, Moustacchi E. Allelism between pso1-1 and rev3-1 mutants and between pso2-1 and snm1 mutants in Saccharomyces cerevisiae. Curr Genet. 1988;13(1):37-40.

45. Lam AF, Krogh BO, Symington LS. Unique and overlapping functions of the Exo1, Mre11 and Pso2 nucleases in DNA repair. DNA Repair (Amst). 2008;7(4):655-62.

46. Robinson NP, McCulloch R, Conway C, Browitt A, Barry JD. Inactivation of Mre11 does not affect VSG gene duplication mediated by homologous recombination in Trypanosoma brucei. J Biol Chem. 2002;277(29):26185-93.

47. Tan KS, Leal ST, Cross GA. Trypanosoma brucei MRE11 is non-essential but influences growth, homologous recombination and DNA double-strand break repair. Mol Biochem Parasitol. 2002;125(1-2):11-21.

48. Zhang XY, Langenick J, Traynor D, Babu MM, Kay RR, Patel KJ. Xpf and not the Fanconi anaemia proteins or Rev3 accounts for the extreme resistance to cisplatin in Dictyostelium discoideum. PLoS Genetics. 2009;5(9):e1000645.

49. Dong H, Nebert DW, Bruford EA, Thompson DC, Joenje H, Vasiliou V. Update of the human and mouse Fanconi anemia genes. Hum Genomics. 2015;9:32.

50. McCulloch R, Barry JD. A role for RAD51 and homologous recombination in Trypanosoma brucei antigenic variation. Genes Dev. 1999;13(21):2875-88.

51. Proudfoot C, McCulloch R. Distinct roles for two RAD51-related genes in Trypanosoma brucei antigenic variation. Nucleic Acids Res. 2005;33(21):6906-19.

52. Hartley CL, McCulloch R. Trypanosoma brucei BRCA2 acts in antigenic variation and has undergone a recent expansion in BRC repeat number that is important during homologous recombination. Mol Microbiol. 2008;68(5):1237-51.

53. Woodward R, Gull K. Timing of nuclear and kinetoplast DNA replication and early morphological events in the cell cycle of Trypanosoma brucei. J Cell Sci. 1990;95(1):49-57.

54. Siegel TN, Hekstra DR, Cross GA. Analysis of the Trypanosoma brucei cell cycle by quantitative DAPI imaging. Mol Biochem Parasitol. 2008;160(2):171-4. 
55. Glover L, Horn D. Trypanosomal histone gammaH2A and the DNA damage response. Mol Biochem Parasitol. 2012;183(1):78-83.

56. Carson CT, Schwartz RA, Stracker TH, Lilley CE, Lee DV, Weitzman MD. The Mre11 complex is required for ATM activation and the G2/M checkpoint. EMBO J. 2003;22(24):6610-20.

57. Limoli CL, Giedzinski E, Bonner WM, Cleaver JE. UV-induced replication arrest in the xeroderma pigmentosum variant leads to DNA double-strand breaks, gamma-H2AX formation, and Mre11 relocalization. Proc Natl Acad Sci U S A. 2002;99(1):233-8.

58. Fernandez-Capetillo, O., Lee, A., Nussenzweig, M. and Nussenzweig, A. (2004) H2AX: the histone guardian of the genome. DNA Repair (Amst). 3, 959-967.

59. Rothkamm K, Barnard S, Moquet J, Ellender M, Rana Z, Burdak-Rothkamm S. DNA damage foci: Meaning and significance. Environ Mol Mutagen. 2015;56(6):491-504.

60. Lavin MF. Ataxia-telangiectasia: from a rare disorder to a paradigm for cell signalling and cancer. Nat Rev Mol Cell Biol. 2008;9(10):759-69.

61. Zha S, Boboila C, Alt FW. Mre11: roles in DNA repair beyond homologous recombination. Nat Struct Mol Biol. 2009;16(8):798-800.

62. Panier S, Durocher D. Push back to respond better: regulatory inhibition of the DNA double-strand break response. Nat Rev Mol Cell Biol. 2013;14(10):661-72.

63. Ward TA, Dudasova Z, Sarkar S, Bhide MR, Vlasakova D, Chovanec M, et al. Components of a Fanconi-like pathway control Pso2-independent DNA interstrand crosslink repair in yeast. PLoS Genetics. 2012;8(8):e1002884.

64. Scharer OD. DNA interstrand crosslinks: natural and drug-induced DNA adducts that induce unique cellular responses. Chembiochem. 2005;6(1):27-32.

65. Grillari J, Katinger H, Voglauer R. Contributions of DNA interstrand cross-links to aging of cells and organisms. Nucleic Acids Res. 2007;35(22):7566-76.

66. Clauson C, Scharer OD, Niedernhofer L. Advances in understanding the complex mechanisms of DNA interstrand cross-link repair. Cold Spring Harb Perspect Biol. 2013;5(10):a012732. d

67. Williams HL, Gottesman ME, Gautier J. The differences between ICL repair during and outside of S phase. Trends Biochem Sci. 2013;38(8):386-93.

68. Muniandy PA, Thapa D, Thazhathveetil AK, Liu ST, Seidman MM. Repair of laserlocalized DNA interstrand cross-links in G1 phase mammalian cells. J Biol Chem. 2009;284(41):27908-17.

69. Wood RD. Mammalian nucleotide excision repair proteins and interstrand crosslink repair. Environ Mol Mutagen. 2010;51(6):520-6.

70. McHugh PJ, Ward TA, Chovanec M. A prototypical Fanconi anemia pathway in lower eukaryotes? Cell cycle. 2012;11(20):3739-44.

71. Grossmann KF, Ward AM, Matkovic ME, Folias AE, Moses RE. S. cerevisiae has three pathways for DNA interstrand crosslink repair. Mutat Res. 2001;487(3-4):73-83.

72. Barber LJ, Ward TA, Hartley JA, McHugh PJ. DNA interstrand cross-link repair in the Saccharomyces cerevisiae cell cycle: overlapping roles for PSO2 (SNM1) with MutS factors and EXO1 during S phase. Mol Cell Biol. 2005;25(6):2297-309.

73. Passos-Silva DG, Rajao MA, Nascimento de Aguiar PH, Vieira-da-Rocha JP, Machado CR, Furtado C. Overview of DNA Repair in Trypanosoma cruzi, Trypanosoma brucei, and Leishmania major. J Nucl Acids. 2010;2010:840768.

74. Munari FM, Revers LF, Cardone JM, Immich BF, Moura DJ, Guecheva TN, et al. Sak1 kinase interacts with Pso2 nuclease in response to DNA damage induced by interstrand crosslink-inducing agents in Saccharomyces cerevisiae. J Photochem Photobiol B: Biology. 2014;130:241-53. 
75. Dudas A, Vlasakova D, Dudasova Z, Gabcova D, Brozmanova J, Chovanec M. Further characterization of the role of Pso2 in the repair of DNA interstrand cross-linkassociated double-strand breaks in Saccharomyces cerevisiae. Neoplasma. 2007;54(3):189-94. P

76. Yan Y, Akhter S, Zhang X, Legerski R. The multifunctional SNM1 gene family: not just nucleases. Future Oncol. 2010;6(6):1015-29.

77. Richie CT, Peterson C, Lu T, Hittelman WN, Carpenter PB, Legerski RJ. hSnm1 colocalizes and physically associates with 53BP1 before and after DNA damage. Mol Cell Biol. 2002;22(24):8635-47.

78. Bayele HK, Eisenthal RS, Towner P. Complementation of a glucose transporter mutant of Schizosaccharomyces pombe by a novel Trypanosoma brucei gene. J Biol Chem. 2000;275(19):14217-22. P

79. Mason JM, Das I, Arlt M, Patel N, Kraftson S, Glover TW, et al. The SNM1B/APOLLO DNA nuclease functions in resolution of replication stress and maintenance of common fragile site stability. Hum Mol Genet. 2013;22(24):4901-13.

80. Hirumi H, Hirumi K. Continuous cultivation of Trypanosoma brucei blood stream forms in a medium containing a low concentration of serum protein without feeder cell layers. J Parasitol. 1989;75(6):985-9. E

81. Genois MM, Paquet ER, Laffitte MC, Maity R, Rodrigue A, Ouellette M, et al. DNA repair pathways in trypanosomatids: from DNA repair to drug resistance. Microbiol Mol Biol Rev. 2014;78(1):40-73. 


\section{Supporting information}

877 S1 Figure: Gene disruption vectors: Construction and their effects on the $T$. brucei genome. (A). Sequences corresponding to $5^{\prime}$ and $3^{\prime}$ untranslated (UTR) or coding (CDS) regions of the target gene were amplified using the specified primer combinations (see Table S3) from T. brucei genomic DNA. The resultant fragments (sizes in bp) were sequentially cloned either side of a resistance cassette that includes a gene encoding for a $T$. brucei selectable marker (hygromycin B phosphotransferase, neomycin phosphotransferase, blasticidin-S-deaminase or puromycin N-acetyltransferase) plus the T. brucei $\beta \alpha$ or $\alpha \beta$ tubulin intergenic repeats (tub IR) required for processing the mRNAs (hatched boxes). The restriction sites used to remove the T. brucei integration cassette from the plasmid backbone are noted. (B-G). Schematic representations of the Tbcsb (B), Tbexol (C), Tbmrel1 (D), Tbsnm1 (E), Tbrad5 (F) and Tbrev3 (G) alleles and the effects of their disruption with DNA fragments containing sequences encoding for neomycin phosphotransferase (neo), hygromycin $\mathrm{B}$ phosphotransferase (hyg), blasticidin-S-deaminase (bla) or puromycin $\mathrm{N}$-acetyltransferase (pac), plus the T. brucei tubulin intergenic elements required for processing their mRNAs (hatched boxes). In each panel, P1 and P2 (in black) correspond to the primers used to generate gene specific amplicons from genomic DNA templates while P3, P4, P5 and P6 correspond to neo, hyg, bla and pac specific primers, respectively: The size of the predicted amplicons, denoted by a dashed line (in kb) is given. P2 and P7 (in grey) correspond to the primers used to generate gene specific amplicons from cDNA templates: The size of the predicted amplicons (in bp) is given. The primer sequences and combinations used for each amplification are listed in Supplementary Tables S2 and S3, respectively.

S2 Figure. Phenotypic analysis of T. brucei null mutants. (A). The cumulative cell density of T. brucei Tbexo1 $\Delta$, Tbmre11 $\Delta$, Tbcsb $\Delta$, Tbrad5 $\Delta$ and Tbrev3 $\Delta$ (dashed line) cultures was followed for 8 days and compared against wild type T. brucei (solid line) cultures grown in parallel. Each data point represents the mean cell density \pm standard deviation from three independent cultures. (B). Cell cycle analysis of T. brucei null mutants. The ratio of DAPIstain kinetoplast $(\mathrm{K})$ and one nuclear $(\mathrm{N})$ genomes in one trypanosomes represents a frequently used marker for the $T$. brucei cell cycle. Phase and DAPI images show cells with a $1 \mathrm{~K} 1 \mathrm{~N}$, $2 \mathrm{~K} 1 \mathrm{~N}$ and $2 \mathrm{~K} 2 \mathrm{~N}$ arrangement which is indicative of the G1/S, G2/M and post $\mathrm{M}$ stages, respectively. The growth shows the relative number of nuclear and mitochondrial genomes structures from asynchronous cultures of wild type, Tbmre $11 \Delta \mathrm{Tb} n m 1 \Delta$ and Tbcsb $\Delta$ parasites. The number of cells analysed per cell line is given above each bar. (C). The susceptibility of T. brucei wild type (solid line) and Tbmre11 $($ dotted line) to hydroxyurea, UV, phleomycin and MMS was assessed. All data points are mean values \pm standard deviations from experiments performed in quadruplicate. (D). The susceptibility of T. bruce $i$ wild type and the various null mutant lines against hydroxyurea (HU), phleomycin (Phleo), methyl methanesulfonate (MMS) and ultraviolet radiation (UV), as judged by their $\mathrm{EC}_{50}$ values, was compared and expressed as a fold difference.

S3 Figure. Susceptibility of $T$. brucei null mutants towards DFMO. Dose response curves and $\mathrm{EC}_{50}$ values (in $\mu \mathrm{M}$ ) of $T$. brucei wild type (solid line) and Tbsnml $\Delta(\mathbf{A})$, Tbexol $\Delta$ (B), Tbmre11 $\Delta$ (C), Tbcsb $\Delta$ (D), Tbrad5 $\Delta$ (E) and Tbrev3 $\Delta$ (F) (dotted line) to DFMO. All data points are mean values \pm standard deviations from experiments performed in quadruplicate.

S4 Figure. Susceptibility of $T$. brucei Tbrad51 and Tbbrca2 null mutants towards mechlorethamine. Dose response curves (A). and $\mathrm{EC}_{50}$ values (in $\left.\mu \mathrm{M}\right)(\mathbf{B})$ of $T$. brucei wild 
mean values \pm standard deviations from experiments performed in quadruplicate. (C). The susceptibility of the T. brucei single and double null mutant lines against mechlorethamine, as judged by their $\mathrm{EC}_{50}$ values, were compared and expressed as a fold difference relative to wild type.

\section{S1 Table. T. brucei lines used in this project.}

\section{S2 Table. Primers used in this study.}

\section{S3 Table. Primers: Function and Combinations.}

\section{Figure Legends}

Figure 1: Sequence analysis of potential trypanosomal ICL repair enzymes. (A). Sequences corresponding to the XPG N-terminal (XPG_N) and XPG_I protein (XPG_I) domains of TbEXO1 (accession number XP_847153) were aligned with the equivalent regions from the $H$. sapiens (HsHEX1; AAC32259) and S. cerevisiae (ScEXO1; NP_014676) orthologues. (B). Sequences corresponding to the HIRAN, SNF2 family N-terminal (SNF2_N; blue box), RING finger (zf-RING_2; purple box) and helicase conserved C-terminal (Helicase_C; red box) domains of TbRAD5 (XP_845733) were aligned with the equivalent regions from the $H$. sapiens (HsHLTF; NP_001305864) and S. cerevisiae (ScRAD5; NP_013132) orthologues. (C). Sequences corresponding to the DNA polymerase family B, exonuclease (DNA_pol_B_exo1), DNA polymerase family B (DNA_pol_B) and C4-type zincfinger of DNA polymerase delta (zf-C4pol) domains, and to the putative REV7 binding site of TbREV3 (XP_847160) were aligned with the equivalent regions from the $H$. sapiens (HsREV3; NP_002903) and S. cerevisiae (ScREV3; CAA97873) orthologues. In the alignments, conserved residues are highlighted in black or grey. (D). Comparison of the Fanconi Anaemia (FA) pathway components expressed by Danio rerio (D. rerio; fish), Drosophila melanogaster (Drosophila; insect), Arabidopsis thaliana (A. thaliana; plant), Saccharomyces cerevisiae (S. cerevisiae; yeast) and T. brucei in relation to the system possessed by humans (H. sapiens). Trypanosomal FA factors were identified by reciprocal BLAST analysis using human Fanconi anemia (FA) factors as bait. An oval indicates the possibility for the presence of a given factor whereas no oval indicates that that component is most probably absent. In mammalian cells, the FA ICL repair pathway can be subdivided the FA core complex (black ovals), recruitment factors (white ovals), FA effectors (grey ovals). In addition to lacking sequences corresponding to the FA core (FANCA, -B, -C, -E, -F, -G, -L and $-\mathrm{T}$ ) or recruitment (FANCD2A and $-\mathrm{I}$ ) complexes, no discernible T. brucei homologues for many of the FA associated proteins (FAAP10, -10, -20, -24 and -100) were detected. Figure layout derived from Dong et al., 2015 [49].

Figure 2: Validation of $T$. brucei null mutant lines. (A-E; upper panels). Amplicons (in kbp) corresponding to intact Tbsnml (A-E), Tbcsb (A), Tbexol (B), Tbmre11 (C), Tbrad5 (D) or Tbrev3 (E) and their hyg-, neo-, pac- or bla-disrupted counterparts were generated from template genomic DNAs extracted from the various T. brucei lines indicated. (A-E; lower panels). Amplicons (in bp) corresponding to intact Tbsnml (A-E), Tbcsb (A), Tbexol (B), Tbmre11 (C), Tbrad5 (D) and Tbrev3 (E) were generated from template cDNAs derived from total RNA extracted from the various $T$. brucei lines indicated. The integrity of cDNAs (and hence RNAs) was evaluated by amplification of a 108 bp control fragment, Tbtert. The primer 
sequences and combinations used for each amplification are listed in S2 and S3 Tables, respectively.

Figure 3: Susceptibility of $T$. brucei null mutants towards mechlorethamine. Left hand panels: Dose response curves and $\mathrm{EC}_{50}$ values (in $\mu \mathrm{M}$ ) of $T$. brucei lines towards mechlorethamine. All data points are mean values \pm standard deviations from experiments performed in quadruplicate. Right hand panels: The susceptibility of the T. brucei single and double null mutant lines against mechlorethamine, as judged by their $\mathrm{EC}_{50}$ values, were compared and expressed as a fold difference relative to wild type.

Figure 4: $\gamma \mathrm{H2A}$ formation in mechlorethamine treated $T$. brucei. (A). T. brucei wild type, Tbsnm1 $\Delta$, Tbcsb $\Delta$, Tbexol $\Delta$ and Tbmre11 $1 \Delta$ were treated with $30 \mu \mathrm{M}$ mechlorethamine. Cell lysates were generated at time intervals $(0,1,2,4,6$, and 8 hours) and analysed by western blot using anti-T. brucei enolase (loading control) and anti-T. brucei $\gamma \mathrm{H} 2 \mathrm{~A}$ antiserum. Treatment with other mechlorethamine concentrations $(10$ and $3 \mu \mathrm{M})$ resulted in similar $\gamma \mathrm{H} 2 \mathrm{~A}$ responses (data not shown). (B). The $\gamma \mathrm{H} 2 \mathrm{~A}$ and enolase signal intensities obtained from western blots containing extracts from three independent mechlorethamine treated cultures were determined using Image Studio ${ }^{\mathrm{TM}}$ Lite (Li-COR Biosciences) and normalised against untreated controls. The data is expressed as a mean \pm standard deviation relative fold difference. $(\mathbf{C})$. The pattern of DNA (blue) and $\gamma \mathrm{H} 2 \mathrm{~A}$ (green) staining in $T$. brucei treated $(+)$ for 4 hours with mechlorethamine (HN2) was compared with signals observed in untreated (-) cells. The cells were examined by florescence microscopy and the brightfield (BF) image captured. Scale bar $=5 \mu \mathrm{M}$.

Figure 5: Evaluating the interplay between TbCSB, TbMRE11 and TbEXO1 in the $T$. brucei ICL repair system. (A-C; upper panels). Amplicons (in kbp) corresponding to Tbcsb $(\mathbf{A} \& \mathbf{B})$, Tbexol $(\mathbf{A} \& \mathbf{C})$ or Tbmrell (B \& C) and their hyg-, neo-, pac- or bla-disrupted counterparts were generated from template genomic DNAs extracted from the various T. bruce $i$ lines indicated. (A-C; lower panels). Amplicons (in bp) corresponding to intact $\mathrm{Tb} c s b$ (A \& B), Tbexol (A \& C) or Tbmrel1 (B \& C) were generated from template cDNAs derived from total RNA extracted from the various $T$. bruce $i$ lines indicated. The integrity of cDNAs (and hence RNAs) was evaluated by amplification of a $108 \mathrm{bp}$ control fragment, Tbtert. The primer sequences and combinations used in for each amplification are listed in S2 and S3 Tables, respectively. (D-F). Dose response curves and $\mathrm{EC}_{50}$ values (in $\mu \mathrm{M}$ ) of $T$. brucei lines towards mechlorethamine are shown. All data points are mean values \pm standard deviations from experiments performed in quadruplicate. The susceptibility of the T. brucei single and double null mutant lines against mechlorethamine, as judged by their $\mathrm{EC}_{50}$ values, were compared and expressed as a fold difference relative to wild type.

Figure 6: Overview of the T. brucei ICL REPAIRtoire. In the T. brucei DNA replicationindependent ICL repair network (A), ICLs (red bar) recognised as a result of global genome surveillance (TbXPC \& TbDDB of GG-NER [41] or stalling of transcription complexes (TbCSB of TC-NER) triggers NER-mediated incision of the DNA backbone, a process that in other organisms involves XPF-ERCC1. This generates an 'unhooked' crosslinked oligonucleotide that undergoes nucleolytic processing (TbSNM1, TbEXO1) resulting in a single nucleotide tethered to the complementary strand, and a single stranded gap. Replication protein A binds to the single stranded DNA gap, promotes activation of PCNA that in turn recruits error free or error prone TLS DNA polymerases which synthesise new DNA (in purple) opposite the unhooked lesion, with the DNA backbone re-joined by DNA ligase: As trypanosomes express multiple TLS DNA polymerases the role of one enzyme in ICL repair 
(e.g. TbREV3) could be complemented by other related activities. A second round of NERmediated incision of the DNA backbone at sites flanking the 'unhooked' crosslinked single nucleotide followed by TLS results in complete removal of the covalent linkage and restoration of dsDNA. In the T. brucei DNA replication-dependent ICL repair network (B), two DNA replication forks converge upon an ICL, causing collapse of each complex. Following recognition (trypanosomal mechanism unknown), incision of the DNA backbone occurs, possibly through the activity of XPF-ERCC1, MUS18 or FAN1 endonucleases, resulting in double strand DNA break (DSB) formation (detected by TbMRE11-mediated formation of $\gamma \mathrm{H} 2 \mathrm{~A}$ ), unhooking of the ICL and generation of a single stranded gap: The unhooked ICL may undergo nucleolytic processing (TbEXO1). A first round of TLS activity results in filling of the single stranded gap associated with formation of the 'unhooked' sequence followed by a second round of NER-mediated incision that removes the ICL from the genome, and TLS that restores the dsDNA structure. The DSBs are then repaired by HR (involving TbRAD51 and TbBRCA2) using the newly formed dsDNA as template. Activities that have been shown to be involved in ICL repair are highlighted in black (XPC, DDB1, SNM1, EXO1, MRE11, RAD51 and BRCA2) or white (CSB) whereas activities that are hypothesised to function in these systems (XPF-ERCC1, XPG, MUS81, FAN1, TLS Pols \& ligase) are in grey. 
A

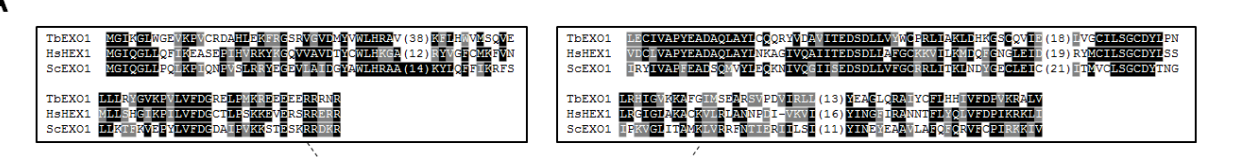

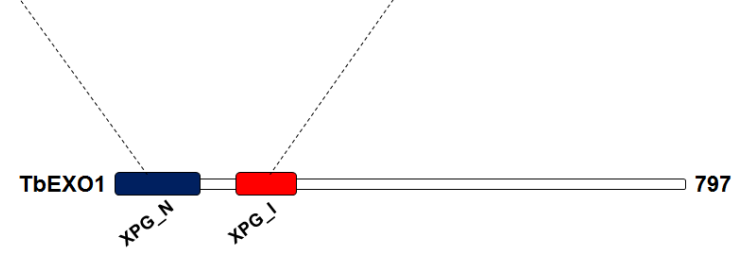

C

Figure 1

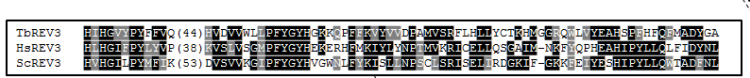

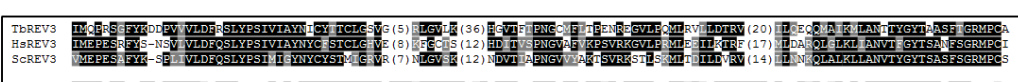

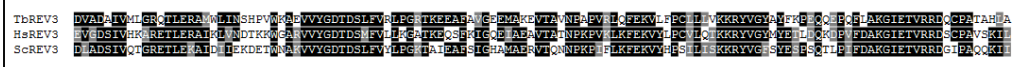

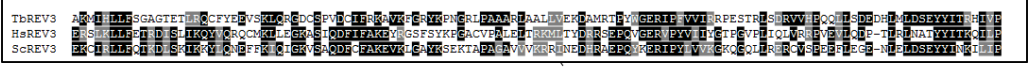

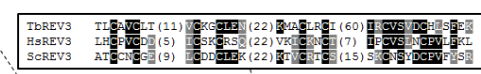

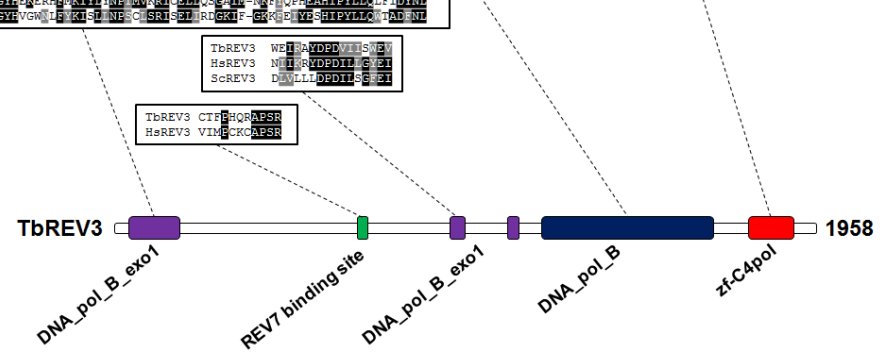

\section{B}

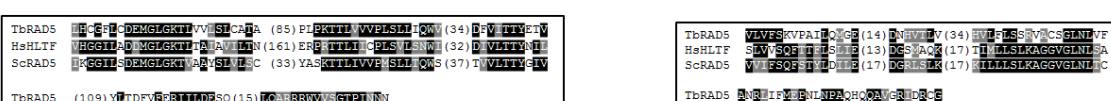

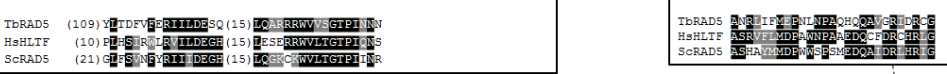

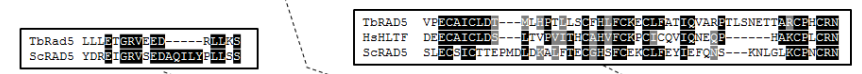

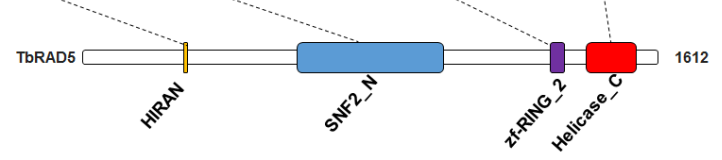

D

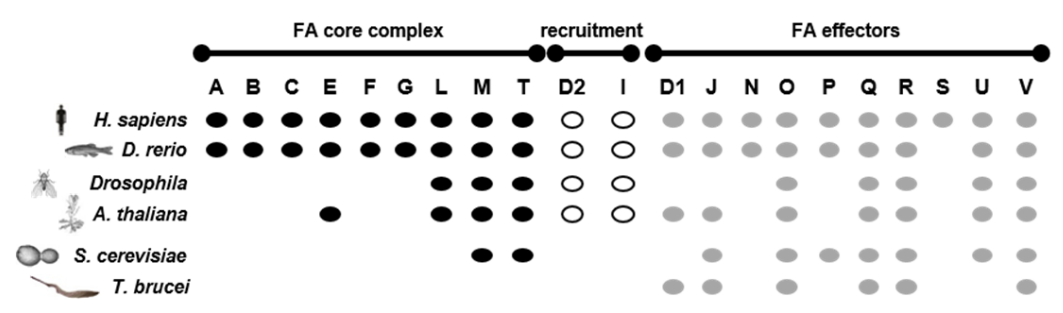


bioRxiv preprint doi: https://doi.org/10.1101/543975; this version posted February 8, 2019. The copyright holder for this preprint (which was not certified by peer review) is the author/funder, who has granted bioRxiv a license to display the preprint in perpetuity. It is made available under aCC-BY 4.0 International license.

1043
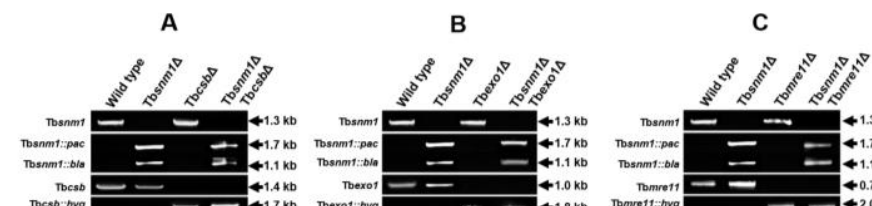

D

E

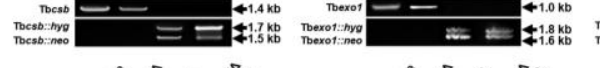

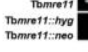
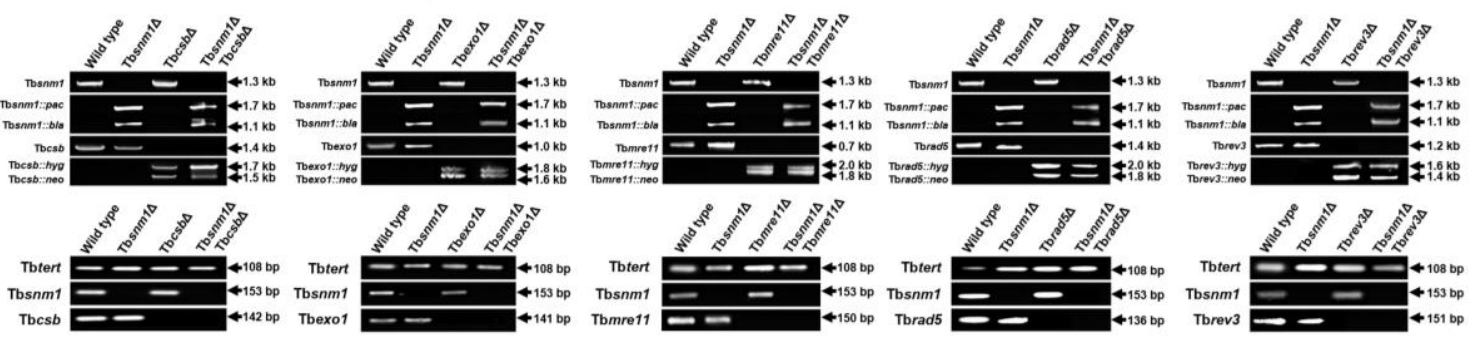

1045

Figure 2 
A

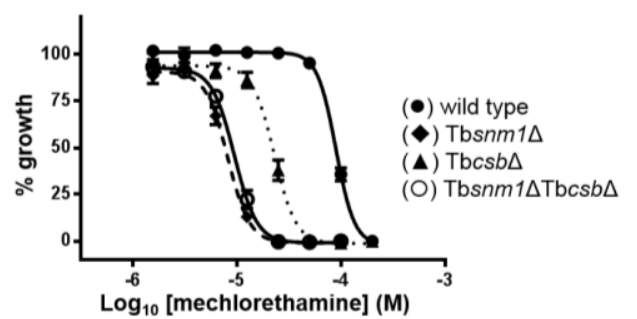

B

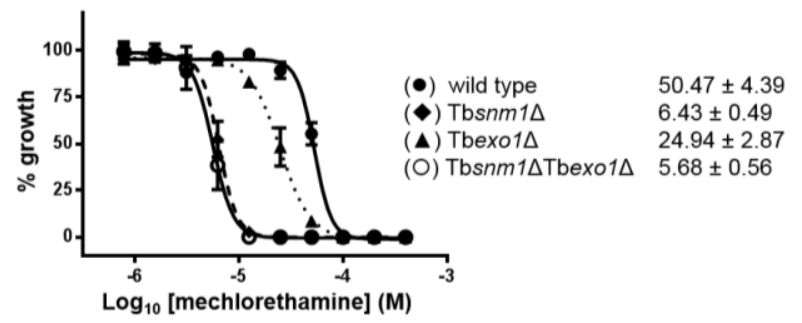

C

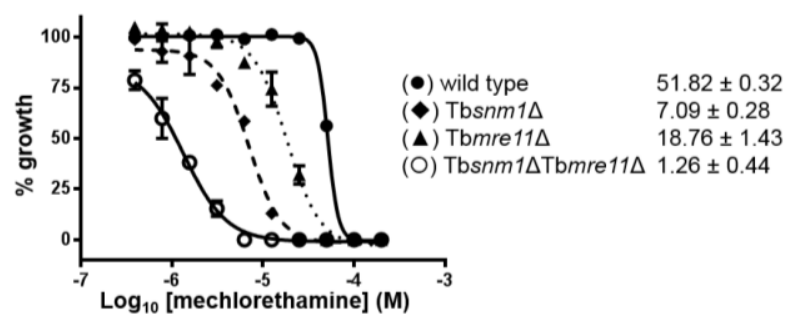

D

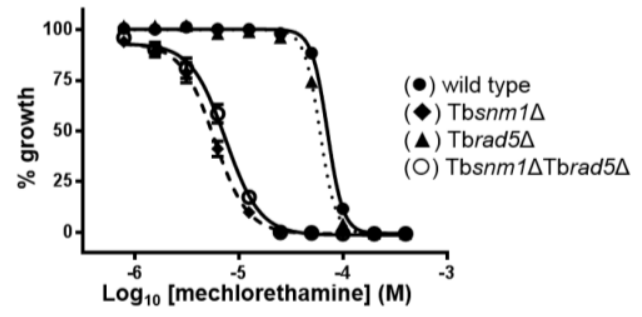

E

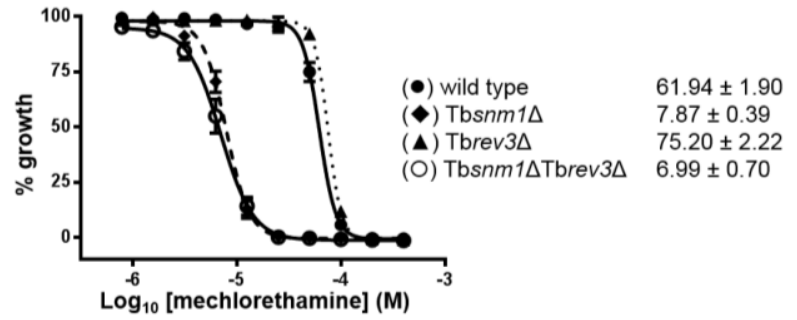

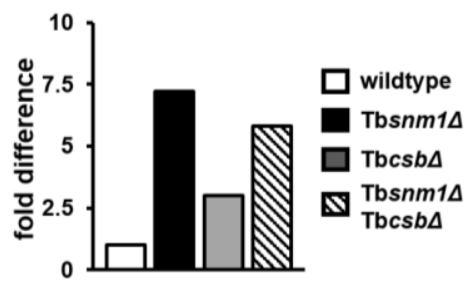
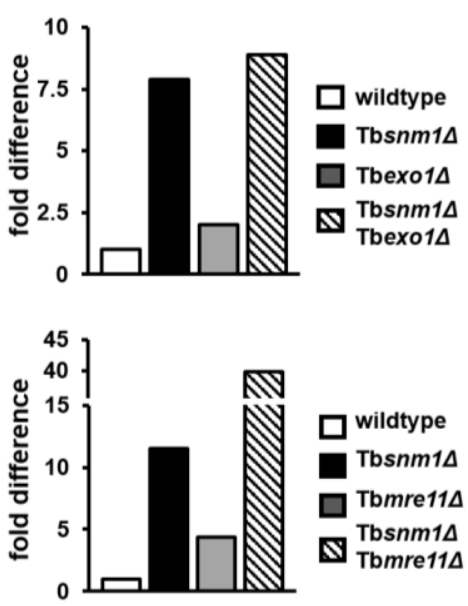

$70.94 \pm 1.25$ $5.76 \pm 0.41$ $60.17 \pm 0.90$ $7.45 \pm 0.21$
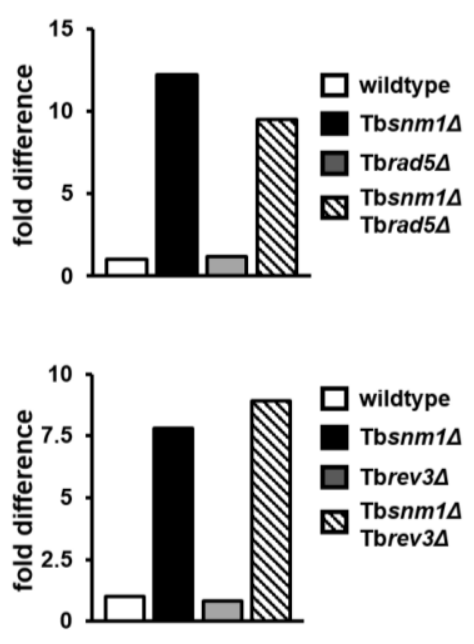

Figure 3 
bioRxiv preprint doi: https://doi.org/101101/543975. this version posted February 8,2019 . The copyright holder for this preprint (which was not certified by peer review) is the author/funder, who has granted bioRxiv a license to display the preprint in perpetuity. It is made available under aCC-BY 4.0 International license.

A
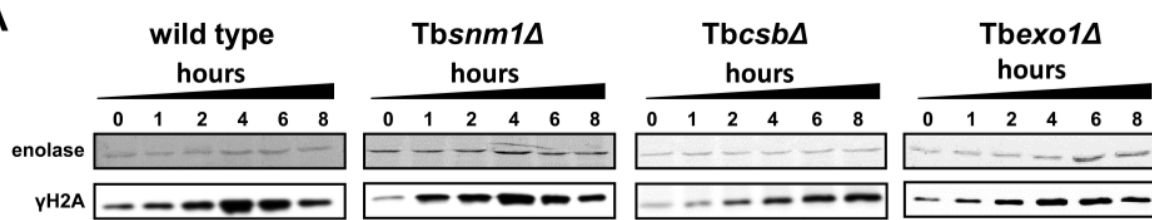

Tbmre11

YH2A
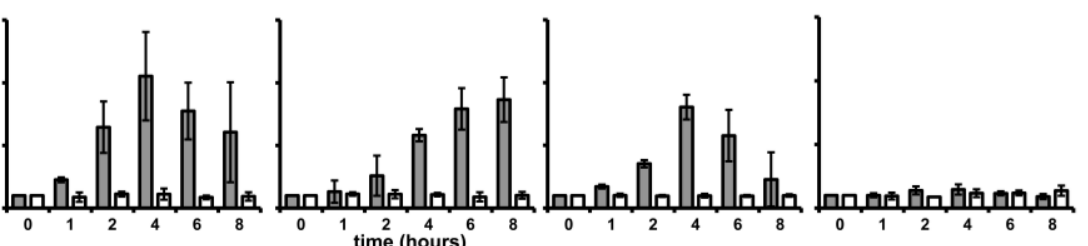

C

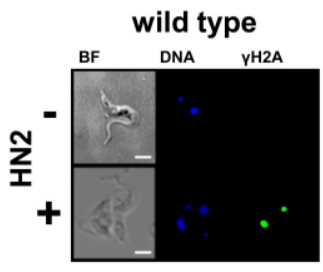

Tbsnm1D

Tbcsbs

Tbex014

Tbmre11
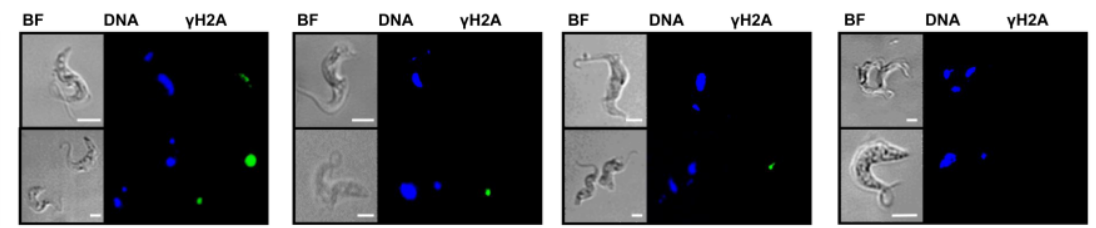

Figure 4 

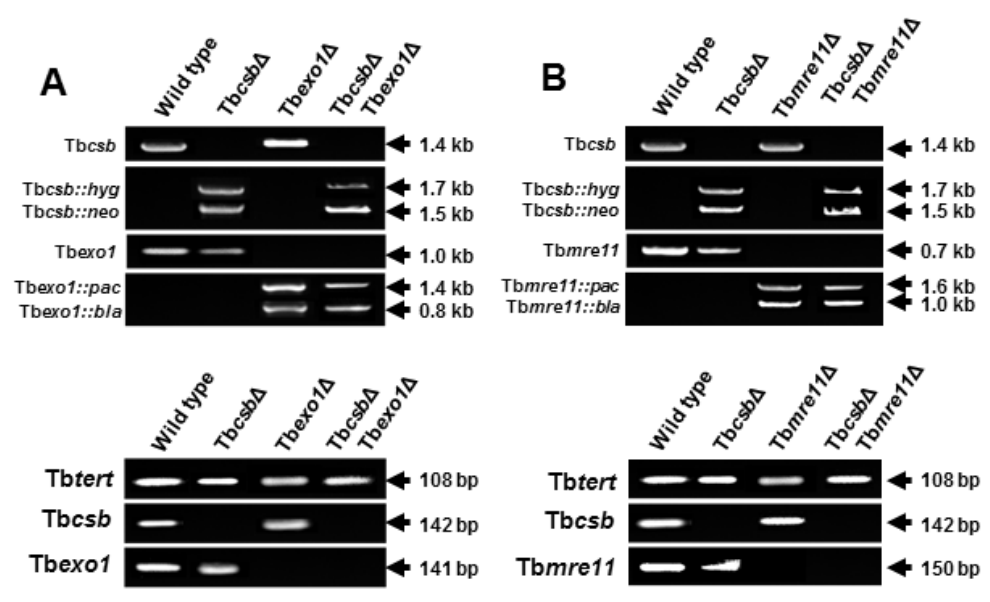
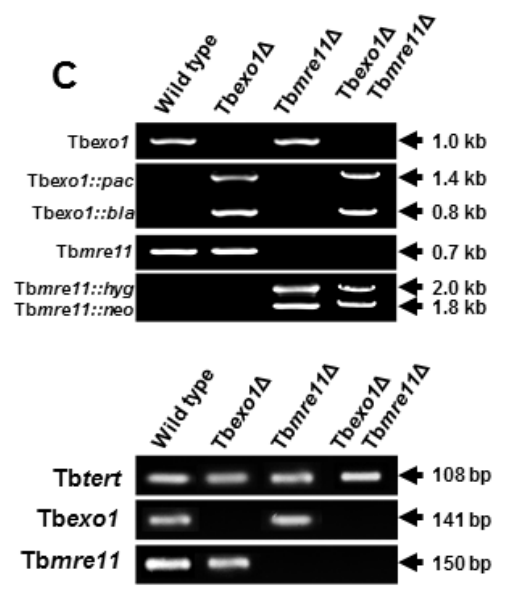

D

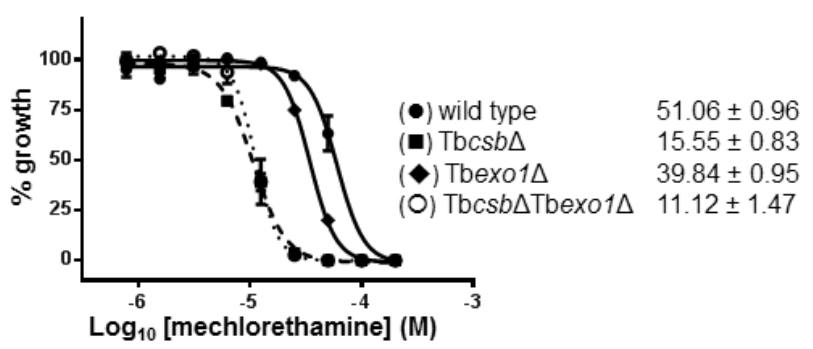

E

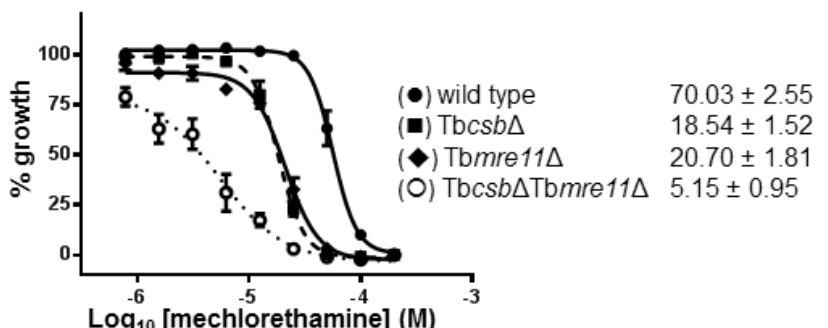

F

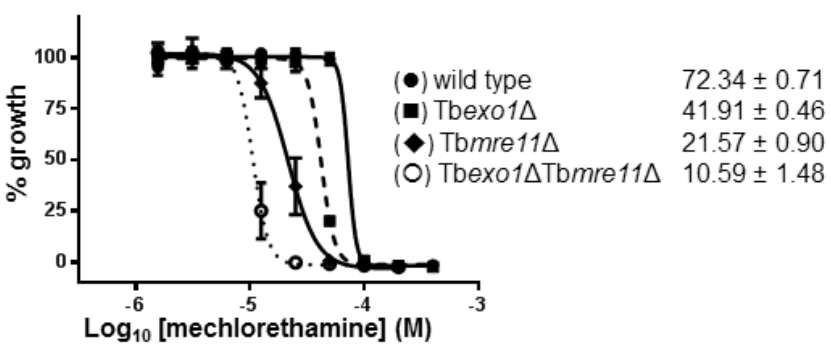

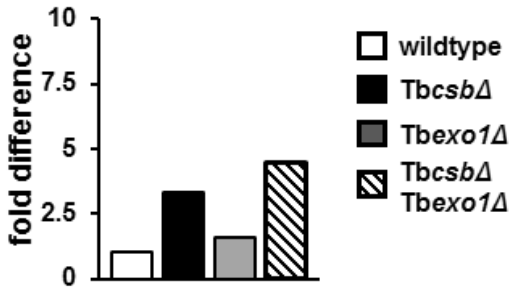
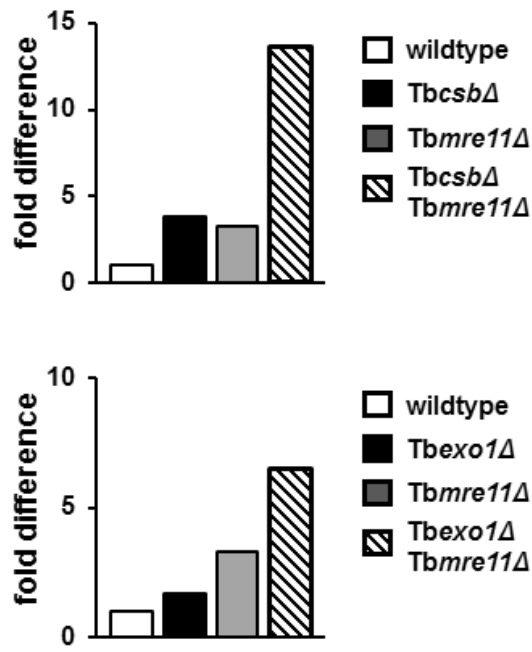

\section{Figure 5}


bioRxiv preprint doi: https://doi.org/10.1101/543975. this version posted February 8, 2019. The copyright holder for this preprint (which was not certified by peer review) is the author/funder, who has granted bioRxiv a license to display the preprint in perpetuity. It is made available under aCC-BY 4.0 International license.

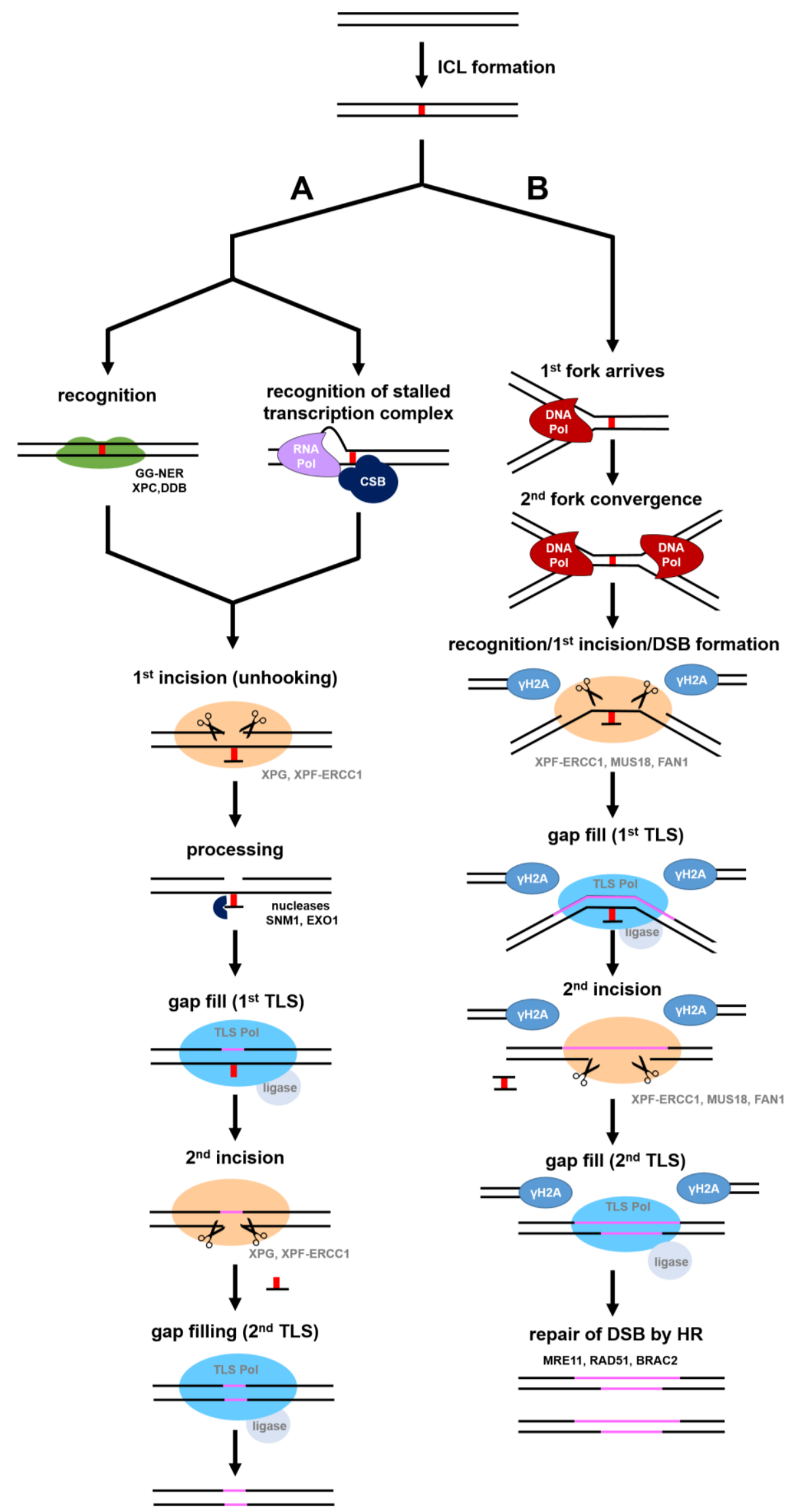

